\title{
A New Formulation of the Initial Value Problem for Nonlocal Theories
}

\author{
Neil Barnaby \\ Canadian Institute for Theoretical Astrophysics, University of Toronto, \\ 60 St. George St., Toronto, ON M5S 3H8, Canada
}

\begin{abstract}
There are a number of reasons to entertain the possibility that locality is violated on microscopic scales, for example through the presence of an infinite series of higher derivatives in the fundamental equations of motion. This type of nonlocality leads to improved UV behaviour, novel cosmological dynamics and is a generic prediction of string theory. On the other hand, fundamentally nonlocal models are fraught with complications, including instabilities and complications in setting up the initial value problem. We study the structure of the initial value problem in an interesting class of nonlocal models. We advocate a novel new formulation wherein the Cauchy surface is "smeared out" over the underlying scale of nonlocality, so that the the usual notion of initial data at $t=0$ is replaced with an "initial function" defined over $-M^{-1} \leq t \leq 0$ where $M$ is the underlying scale of nonlocality. Focusing on some specific examples from string theory and cosmology, we show that this mathematical re-formulation has surprising implications for the well-known stability problem. For D-brane decay in a linear dilaton background, we are able to show that the unstable directions in phase space cannot be accessed starting from a physically sensible initial function. Previous examples of unstable solutions in this model therefore correspond to unphysical initial conditions, an observation which is obfuscated in the old formulation of the initial value problem. We also discuss implication of this approach for nonlocal cosmological models.
\end{abstract}

Keywords: nonlocal field theory, string field theory, tachyon condensation, delay differential equations

\section{Introduction}

Nearly all dynamical systems encountered in physics are local. Time evolution is usually described by differential equations involving at most two time derivatives, and the $t>0$ dynamics depend only on a small amount of data

Email address: barnaby@cita.utoronto.ca (Neil Barnaby) 
specifying the state of the system at $t=0$. There are, however, a number of reasons to entertain the possibility that locality is violated on microscopic scales, for example through the appearance of an infinite series of higher derivatives in the fundamental equations of motion. This type of nonlocality is a generic feature of many particle physics models including string theory [1], field theories living on noncommutative space-time [2] and also models with a minimal length scale [3]. In fact, it has been argued that any Quantum Field Theory (QFT) of gravity must be nonlocal at the most fundamental level [4].

The underlying nonlocality of string theory manifests itself strikingly through the infinite derivative structure of String Field Theory [5] (SFT) and also toy models such as the $p$-adic string [6, 7] or strings quantized on a random lattice [8]. These constructions make it possible to study both nonperturbative and off-shell string physics, and have therefore played a key role in describing the decay of unstable D-brane configurations (tachyon condensation). The dynamics of tachyon condensation [9] are relevant for both formal string theory and also cosmology, because brane/anti-brane annihilation [10] marks the endpoint of popular D-brane inflation models [1].

Aside from their "bottom up" motivations, field theories with infinitely many derivatives are interesting due to improved Ultra-Violet (UV) behaviour [12, 13] and novel cosmological dynamics [14, 15, 16, 17, 18, 19, 20, 21, 22, 23]. In [21, 22] it was shown that nonlocal theories can support inflation even on a very steep potential, alleviating the fine tuning problems that plague inflationary model building. This mechanism leads to a robust prediction for large nongaussianity in the Cosmic Microwave Background (CMB) which will be falsifiable (or verifiable!) with Planck [22, 23].

In spite of these motivations, physical applications of nonlocal field theories are fraught with complications. Perhaps the most serious concerns are the following.

1. Stability: If the equation of motion admits more than two initial data then the "extra" degrees of freedom can be interpreted as physical excitations which carry wrong-sign kinetic energy and render the Hamiltonian unbounded from below [4, 12, 24, 25]. The quantum theory contains ghosts, while the classical theory is plagued by Ostrogradski (higher derivative) instabilities.

2. Predictivity: If the equation of motion requires infinitely many initial data, then there is an independent concern. By suitable choice of the infinite free parameters of the solution it might be possible to arrange for nearly any time dependence over an arbitrarily long interval. In this case the initial value problem would be completely bereft of predictivity. (Throughout this paper we understand the word "predictivity" in this sense.)

Clearly, very much is at stake. There is evidence that both of these problems might manifest themselves in string theory [4], for example through the wild oscillatory dynamics of tachyon condensation in flat space [7] (however, see [26]). 
As emphasized in 24], the presence of ghost-like instabilities in a nonlocal QFT is intimately related to the structure of the initial value problem for the classical equations of motion. In this work, we consider a new formulation of the initial value problem for a broad class of nonlocal theories which incorporate dissipation [27, 28, 29] into the dynamics (as is unavoidable in a cosmological context). We show that, in such models, the infinite tower of initial conditions $\partial_{t}^{(n)} \phi$ at $t=0$ can be swapped for the freedom to fix the time evolution over some interval $\Delta t=T$ by requiring

$$
\phi(t)=\phi_{0}(t) \quad \text { on } \quad t \in[-T, 0]
$$

with $\phi_{0}(t)$ the "initial function" and $T^{-1}$ related to the energy scale at which nonlocal effects become important. This new formulation has considerable intuitive appeal since it makes manifest the nonlocal structure of the theory; the Cauchy surface has been "smeared out" over a finite interval set by the underlying scale of nonlocality.

This mathematical re-formulation of the initial value problem suggests a rather novel new perspective on the key problems of predictivity and stability discussed above. Although the initial function is arbitrary from a purely mathematical viewpoint, we point out that $\phi_{0}(t)$ must be subjected to some auxiliary constraints in order to ensure that the $-T \leq t \leq 0$ evolution is physically sensible. For example, in the case of D-brane decay $\phi_{0}(t)$ should be restricted to the physically meaningful region of the potential. The physical interpretation of these constraints is completely obfuscated in the "old" formulation of the initial value problem wherein one fixes infinitely many derivatives at $t=0$.

Remarkably, these mild physical restrictions on $\phi_{0}(t)$ have the effect of constraining the theory to a stable subset of its solution space. Although the Hamiltonian is globally unbounded below, the unstable directions in phase space cannot be accessed from an allowed initial configuration. This restriction is nonperturbative and retains information about the full nonlocal structure of the equations. Moreover, the constrained theory makes robust predictions for the macroscopic $(\Delta t \gg T)$ dynamics.

In light of these results, we argue that there exists a broad class of stable, predictive nonlocal models whose equations of motion admit infinitely many initial data. A specific example is provided by D-brane decay in the background of a linear dilaton [27, 28, 29]. Previous examples of unstable solutions in this model were implicitly assuming unphysical initial conditions. This perspective on the nature of the instability was not appreciated in previous studies because the initial value problem was formulated in a highly unnatural way.

Our results help to establish the theoretical consistency of nonlocal cosmological models, and also provides a plausible mechanism by which the consistent inclusion of closed strings might enable SFT to evade the Ostrogradski instability. (This has been conjectured as a possible solution of the instability problems that plague SFT, however, to our knowledge no concrete mechanism has previously been proposed.) Since we do not rely on any intrinsically stringy properties of the theory, it will be straightforward to apply our approach to more general 
nonlocal models, in cosmology or elsewhere. At the practical level, our analysis provides a new tool-box of efficient and numerically stable techniques for solving nonlinear equations with infinitely many derivatives.

The organization of this paper is as follows. In section 2 we discuss some general properties of delayed differential equations which will be important for the subsequent analysis. In section 3 we re-visit models of light-like D-brane decay in light of the formulation (1) of the initial value problem, showing how macroscopic predictivity and stability can be salvaged. In section 4 we show how the simple-minded inclusion of dissipation can lead to delay-type nonlocality in a very general nonlocal QFT framework and we show how the formulation (1) of the initial value problem can be implemented. In section 5 we study a particular model, cosmological D-brane decay, showing again how stability/predictivity can be salvaged. Finally, in section 6, we conclude.

\section{Nonlocal Models with Delays}

In this section we will provide a brief discussion of some general features of models whose nonlocality arises purely through delay operators of the form $e^{-T \partial_{t}}$. As we will see shortly, this type of nonlocality is often associated with the presence of some source of dissipation in the dynamics (such as cosmological friction).

\subsection{The Method of Steps}

Consider a prototype model whose dynamics are governed by the following delay differential equation

$$
\sum_{n=0}^{N} a_{n} \partial_{t}^{(n)} \phi(t)=F[\phi(t-T)]
$$

Here $F[x]$ is some nonlinear forcing term and we assume that $T>0$. Equation (2) is a nonlinear differential equation of infinite order, which is easy to see by noting the identity $\phi(t-T)=e^{-T \partial_{T}} \phi(t)=\sum_{n} \frac{(-T)^{n}}{n !} \partial_{t}^{(n)} \phi(t)$. Infinite order equations have been studied in some detail using the formal generatrix calculus in 24] and also [25, 31]. (See [32, 33, 34, 35] for different approaches.)

The model (2) belongs to a special class of nonlocal theories, referred to as having "compact support" in [4], which can be formulated in a surprisingly simple and intuitive manner using the method of steps (see also [29]). The key observation is that equation (2) defines a mapping from functions on the interval $[t-T, t]$ to the interval $[t, t+T]$. We can therefore break the solution $\phi(t)$ into segments $\phi_{i}(t)$ defined over contiguous intervals of length $T$. Explicitly, we set

$$
\phi(t)=\phi_{i}(t) \quad \text { on } \quad t \in[(i-1) T, i T]
$$

where the integer $i=0,1,2, \cdots$ labels the segment under consideration. This procedure for splitting a solution up into segments is illustrated schematically 
in Fig. 1. The dynamical equation (2) provides a recursion relation to obtain the $i$-th solution segment:

$$
\sum_{n=0}^{N} a_{n} \partial_{t}^{(n)} \phi_{i}(t)=F\left[\phi_{i-1}(t-T)\right], \quad i=1,2, \cdots
$$

On the first segment $(i=0)$ we have an arbitrary "initial function" $\phi_{0}(t)$. More on this shortly.

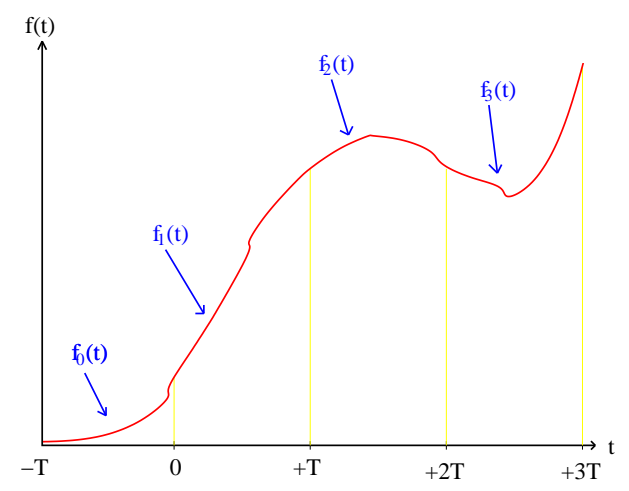

Figure 1: Schematic illustration of the procedure for splitting a function up into solution segments defined over contiguous intervals of length $\Delta t=T$.

Starting from a given $\phi_{0}(t)$, the stepping procedure (4) is very easy to implement. Each solution segment is obtained by solving an inhomogeneous linear differential equation whose source term is constructed from the previous solution segment. At the points $t=i T$ where the segments are joined we should impose boundary conditions that $\phi(t)$ be continuous and as smooth as possible. Since the differential operator on the left-hand-side of (4) is $N$-th order, we can demand that

$$
\left.\partial_{t}^{(n)} \phi_{i+1}(t)\right|_{t=i T}=\left.\partial_{t}^{(n)} \phi_{i}(t)\right|_{t=i T} \quad \text { for } \quad n=0, \cdots, N-1
$$

This matching should be performed at each knot: $i=0,1, \cdots$ The method of steps is nonperturbative and solutions obtained using the recursion (4) are often much more efficient and numerically stable than those obtained using alternative approaches (such as higher order perturbation theory [24] or the diffusion equation technique [18]).

Notice that, in general, the $N$-th derivative of $\phi(t)$ will be discontinuous at $t=0$. This discontinuity will propagate into higher derivatives in subsequent segments. The only way to avoid this effect is by choosing $\phi_{0}(t)$ to be a solution of (2). In practice, however, we can minimize derivative discontinuities by using perturbation theory to construct the initial function. None of our qualitative results in this work will rely on the smoothness properties of the solutions in any way. 


\subsection{The Initial Value Problem}

The initial value problem associated with (2) can be formulated as

$$
\phi(t)=\phi_{0}(t) \quad \text { on } \quad t \in[-T, 0]
$$

where the initial function $\phi_{0}(t)$ specifies the state of the system on the first segment. Equation (6) can be regarded as "smearing out" the Cauchy surface over a finite width. Notice that (6) is not the unique formulation of the initial value problem for this model. If we have adopted perturbation theory (instead of the method of steps) it would have been most natural to specify the derivatives $\partial_{t}^{(n)} \phi$ at $t=0$, following [24]. Although both views of the initial value problem are mathematically valid, the formulation (6) is much more natural here because it makes the manifest the delay-type nonlocality of the underlying theory.

The initial value problem (6) is unusual. The conventional notion of causality 1 is violated, because the evolution depends on past history. Suppose that we are observing the dynamics of $\phi(t)$ over a time scale short compared to the delay, $\Delta t \ll T$. Equations (2) and (6) imply that the system will be directly influenced by events which occurred very far in the past, before the start of the "experiment". No finite amount of information about the state of the system at $t=0$ is sufficient to uniquely determine the $t>0$ evolution. Indeed, equation (6) means that we could arrange for any time dependence over an interval $\Delta t=T$, regardless of the specific details of the dynamical equation (2). Clearly, the nonlocality of the system is associated with a breakdown of predictivity over microscopic scales, $\Delta t \ll T$.

On the other hand, if we observe the system for a macroscopic time scale, $\Delta t \gg T$, then the situation is different. We retain a weakened version of causality because the "memory" is small as compared to the dynamical scales of interest. We could hope to measure the evolution over an entire segment and use this as an initial function to robustly predict the subsequent dynamics. (In principle this still requires making infinitely many measurements and, in a realistic model, it might only be possible to reconstruct the initial function to some limited accuracy. Any uncertainty in $\phi_{0}(t)$ propagates into all future predictions. In practice, this effect is usually tiny because the late-time dynamics are insensitive to the details of the initial function.)

Notice that, although the initial function may be ambiguous in some circumstances, it is not completely arbitrary. We should subject $\phi_{0}(t)$ to some auxiliary constraints in order to ensure that the $-T \leq t \leq 0$ evolution was sensible. The specific nature of these constraints will, obviously, depend on the system that we are modeling. Notice that we have already seen an example of this necessity: arbitrary choices of $\phi_{0}(t)$ will lead to derivative discontinuities. We will see shortly how the imposition of physical restrictions on $\phi_{0}(t)$ can have very significant implications for the $t>0$ dynamics of the system.

\footnotetext{
${ }^{1}$ For our purposes, the conventional notion of causality can be caricatured as "the present determines the future".
} 


\section{Delayed Dynamics in String Theory}

In the last section we introduced a general class of models with delay-type nonlocality, equation (2). We argued that (6) provides the most natural formulation of the initial value problem. Let us now consider two explicit examples from string theory and show how this new perspective on the initial value problem provides invaluable insight into the key problems - stability and predictivity - discussed in the introduction.

\subsection{An Example from p-adic String Theory}

Let us now consider an explicit example of the type (2) whose nonlinear dynamics are particularly transparent. Our example is based on $p$-adic string theory [6], a toy model of the bosonic string which is known to reproduce a number of key features of the full string theory. (See 36] for a review of some of these features and [36, 37] for a discussion of the $p$-adic theory at finite temperature and implications for the cosmological constant problem.) The $p$ adic theory contains a single scalar field $\phi(x)$, the open string tachyon, that encodes the instability of a space-filling D-brane. Following 27] we adopt lightcone coordinates $x^{ \pm}=\frac{1}{\sqrt{2}}\left(x^{0} \pm x^{1}\right)$ so that the metric takes the form $d s^{2}=$ $-\left(d x^{0}\right)^{2}+\left(d x^{1}\right)^{2}=-2 d x^{+} d x^{-}$and assume a linear dilaton profile: $\Phi(x)=$ $-V^{+} x^{-}$. In this closed string background the $p$-adic Lagrangian is [27]

$$
\mathcal{L}=\frac{e^{V^{+} x^{-}}}{g_{p}^{2}}\left[-\frac{1}{2}\left(p^{-\frac{1}{2} \square} \phi\right)^{2}+\frac{1}{p+1} \phi^{p+1}\right]
$$

where $p$ is a prime number, $g_{p}$ is related to the open string coupling, we have set $\alpha^{\prime} \equiv m_{s}^{-2} \equiv 1$. The potential is

$$
g_{p}^{2} e^{-V^{+} x^{-}} V(\phi)=+\frac{1}{2} \phi^{2}-\frac{1}{p+1} \phi^{p+1}
$$

which is plotted in Fig. 2, If we assume a light-like tachyon profile $\phi=\phi\left(x^{+}\right)$ then the equation of motion derived from (7) takes a remarkably simple form

$$
\phi\left(x^{+}\right)=\phi^{p}\left(x^{+}-T\right)
$$

where the delay is

$$
T \equiv V^{+} \ln p
$$

Equation (9) admits two constant solutions: $\phi=0$ and $\phi=1$ (also $\phi=-1$ when $p$ is odd) corresponding, respectively, to the local maxima and minima of the potential. Physically, the solution $\phi=1$ corresponds to state with an unstable space-filling D-brane while $\phi=0$ is the empty state containing neither D-brane nor open string excitations 2 The dynamical process of rolling the

\footnotetext{
${ }^{2}$ This configuration is sometimes called the "vacuum" although, properly, the theory (7) has no minimum energy state.
} 


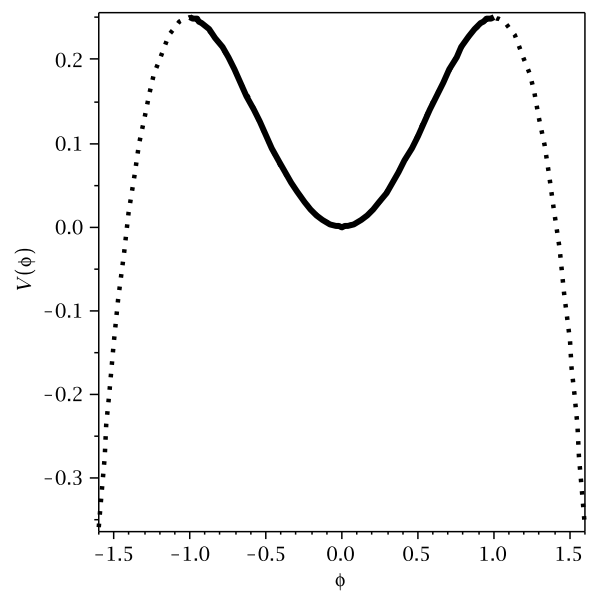

Figure 2: The potential for the $p$-adic tachyon field $\phi$ taking $p=3$ for illustration. The maxima $\phi= \pm 1$ correspond to the physical state containing an unstable D-brane while the minimum at $\phi=0$ is the "vacuum" containing neither D-brane nor open string excitations. The unbounded regions are thought to be associated with the closed string tachyon. The solid part of the potential, $-1<\phi<+1$, denotes the range of field values which can be accessed by physically meaningful initial functions.

tachyon from the unstable maximum to the minimum of the potential - tachyon condensation - provides a time-dependent description of D-brane decay in the $p$-adic string theory.

The initial value problem (6) requires that we choose the evolution of the $p$-adic tachyon over a time interval $\Delta x^{+}=T=V^{+} \ln p$. Which choices of $\phi_{0}\left(x^{+}\right)$will lead to a sensible physical interpretation in terms of brane decay? To answer this question, first notice that the potential (8) is unbounded below for $\phi>1$ (also $\phi<-1$ for $p$ odd). This is usually thought to be associated with the presence of the closed string tachyon [39] and solutions rolling down the unbounded region are regarded as unphysical. Second, recall that near $\phi=0$ there are no open string excitations (because there is no brane for them to end on) and hence the physical meaning of the open string variable $\phi$ is obscure. We should therefore expect that the theory (7) only admits a robust physical interpretation in terms of brane decay when the initial function satisfies:

$$
0<\phi_{0}\left(x^{+}\right)<1
$$

When $p$ is odd we can continue this to $-1<\phi_{0}\left(x^{+}\right)<0$. Thus, only the solid region of the potential curve plotted in Fig. 2 can be accessed by physically meaningful initial functions.

Equation (9) can be easily solved using the method of steps. However, it happens that we can write down a simple exact solution which is valid on all segments, as was pointed out in [28]. That solution is

$$
\phi\left(x^{+}\right)=\exp \left[-e^{x^{+} / V^{+}} F\left(x^{+}\right)\right]
$$


where $F\left(x^{+}\right)$is a periodic function satisfying

$$
F\left(x^{+}\right)=F\left(x^{+}-T\right)
$$

(The solution (12) is always positive, however, when $p$ is odd we can obtain a second solution simply by flipping the sign.) The initial value problem (6) fixes the free function $F\left(x^{+}\right)$on the first segment as

$$
F\left(x^{+}\right)=-e^{-x^{+} / V^{+}} \ln \left[\phi_{0}\left(x^{+}\right)\right] \quad \text { on } \quad t \in[-T, 0]
$$

The periodicity relation (13) then uniquely determines $F\left(x^{+}\right)$on all subsequent segments.

Armed with the exact closed-form solution (12) we can address the nonlinear dynamics of (7) is a remarkably simple way. If the initial function satisfies (11) then we must have $F\left(x^{+}\right)>0$ and the solution (12) approaches the minimum $\phi=0$ very rapidly for $t \gtrsim T$. This behaviour is illustrated in the left panel of Fig. 3. Notice the peculiar dynamics: $\phi\left(x^{+}\right)$can roll to higher potential energy during the rolling, even though the dynamics are governed by gradient flow. Such motion is possible because the kinetic energy in this theory can be negative. Nevertheless, the nonlinear dynamics are stable in the sense that the field does eventually settle down to the minimum of the potential at late times. Note also that these late-time asymptotics are largely insensitive to details of $\phi_{0}\left(x^{+}\right)$.

The Hamiltonian associated with (7) is unbounded from below 28]. However, we have just argued that the nonlinear solutions of this theory do not display any unstable behaviour. How can this be? To understand what is going on, we should imagine relaxing the constraint (11) on the initial function. Now the dynamics associated with (9) are very different. If $\phi_{0}\left(x^{+}\right)>1$ anywhere on $x^{+} \in[-T, 0]$ then we must have $F\left(x^{+}\right)<0$ at one or more points and consequently the solution (12) undergoes wild oscillations with divergent amplitude. This is a manifestation of the Ostrogradski instability. These dynamics are illustrated in the right panel of Fig. 3. As can be seen, the qualitative late-time behaviour is now highly sensitive to the details of $\phi_{0}\left(x^{+}\right)$.

In the formulation (6) it is quite apparent what is going wrong with the solutions plotted in the right panel of Fig. 3. We have allowed the tachyon to sample unphysical regions of the potential on the initial segment $-T \leq t \leq$ 0 . However, this conclusion would be completely obfuscated if we adopted a perturbative approach. Writing $\phi\left(x^{+}\right)=1+\delta \phi\left(x^{+}\right)$we obtain

$$
\left[p^{V^{+} \partial_{+}}-p\right] \delta \phi\left(x^{+}\right)=0
$$

to linear order in $\delta \phi$. The solution of (15) is

$$
\delta \phi\left(x^{+}\right)=\sum_{n=-\infty}^{+\infty} \alpha_{n} e^{M_{n} x^{+}}
$$



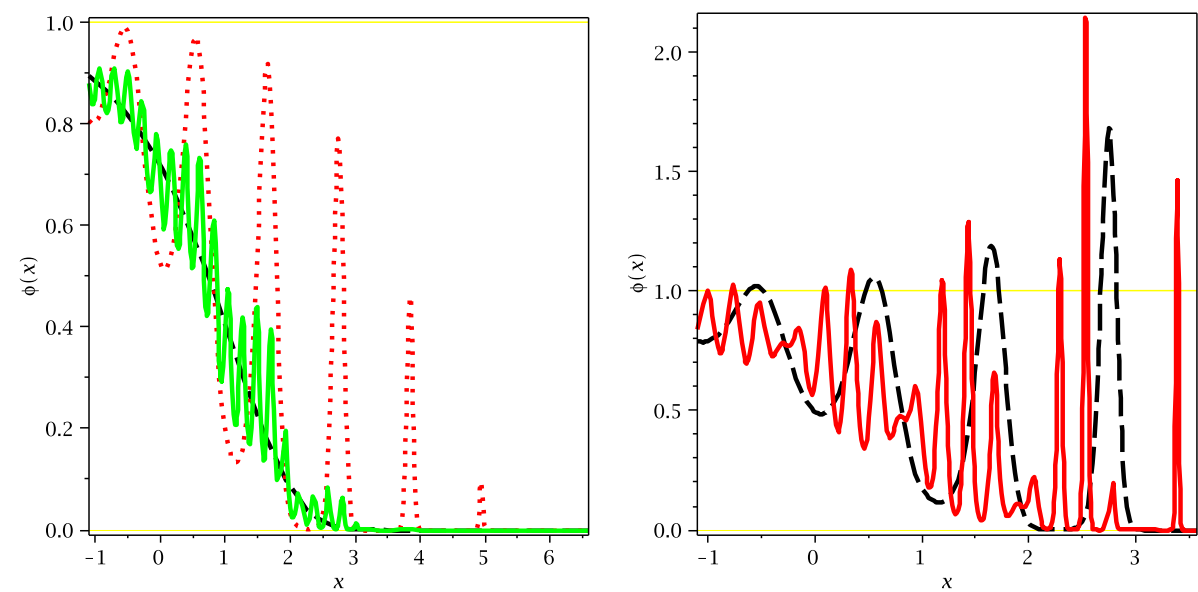

Figure 3: The time evolution of the $p$-adic tachyon field for several representative choices of the initial function. In the left panel we consider only examples which satisfy the constraint $0<\phi_{0}\left(x^{+}\right)<1$. At late times the field settles down to the minimum of the potential and the macroscopic $\left(\Delta x^{+} \gg T\right)$ dynamics are largely insensitive to the details of $\phi_{0}\left(x^{+}\right)$. In the right panel we consider two examples where the initial function violates the physical constraint $0<\phi_{0}\left(x^{+}\right)<1$. Now the field undergoes erratic oscillatory behaviour at late times and the dynamics become highly sensitive to the details of $\phi_{0}\left(x^{+}\right)$. In both panels we set $p=3$ and $V^{+}=1$ for illustration. The horizontal yellow lines illustrate the critical values $\phi=0$ and $\phi=1$. 
which depends on infinitely many free parameters $\alpha_{n}$. The frequencies $M_{n}$ correspond to the zeroes of the generatix $f(s)=p^{V^{+} s}-p$. Explicitly, we have

$$
M_{n}=\frac{1}{V^{+}}\left[1+\frac{2 \pi i n}{\ln p}\right], \quad n=0, \pm 1, \pm 2, \cdots
$$

The spectrum (17) can be interpreted as a single growing mode (the usual tachyon) along with an infinite tower of modes which oscillate with ever-growing amplitude 3 Half of those spurious modes carry wrong-sign kinetic energy and represent ghost-like excitations [28]. In 28] it was noted that there exists an "island of stability" in initial condition space. By carefully tuning the coefficients $\alpha_{n}$ in the solution (16) we can have sensible late-time dynamics. This tuning looks highly contrived in the perturbative approach, however, in light of the method of steps we see that it amounts to simply satisfying the physical bound (11) over a microscopic time $\Delta x^{+}=T$.

Before moving on, it is worth commenting on a special class of initial functions which we have excluded from our discussion up to now. In the special case where $\phi_{0}\left(x^{+}\right)=1$ for some points on $x^{+} \in[-T, 0]$, then sustained oscillations are possible. At late times the tachyon interpolates periodically between the maximum and minimum, as is illustrated in Fig. 4. Taken at face value, this solution describes a brane which repeatedly dissolves into tachyon matter and then re-assembles itself. We are tempted to interpret this as an array of space-like brane (S-brane) [38] in the $p$-adic string theory. For the time being, however, we remain agnostic as to whether the upper limit $\phi_{0}\left(x^{+}\right)=1$ should be included in the physical constraint (11).

The oscillatory behaviour in Fig. 4 might seem very surprising. The dynamics of the theory (77) is purely dissipative. How can this be reconciled with the observation that solutions need not limit to $\phi=0$ at late times? The key to this puzzle is noting that the dilaton profile $\Phi(x)$ is nearly constant on very small scales $\Delta x^{+} \ll T \sim V^{+}$. Deep in the UV the dissipative effects associated with the dilaton gradient must be negligible. This observation is related to the fact that the spectrum (17) contains frequencies $M_{n}$ which are arbitrarily large as compared to the energy scale associated with the damping, $T^{-1}$.

\subsection{An Example from String Field Theory}

In this subsection we consider a somewhat more realistic example: the level zero truncation of bosonic SFT. In order to obtain a delayed formulation we

\footnotetext{
${ }^{3}$ The solution (16) is completely consistent with (12). This is easily seen by expanding $F\left(x^{+}\right)$in a Fourier series as

$$
F\left(x^{+}\right)=a_{0}+\sum_{n=1}^{\infty} a_{n} \cos \left(\frac{2 \pi n x^{+}}{T}\right)+\sum_{n=1}^{\infty} b_{n} \sin \left(\frac{2 \pi n x^{+}}{T}\right)
$$

When $1 \gg\left|a_{n}\right|,\left|b_{n}\right|$ we can expand (12) in a Taylor series and trivially recover $\phi\left(x^{+}\right) \cong$ $1+\sum_{n} \alpha_{n} e^{M_{n} x^{+}}$.
} 


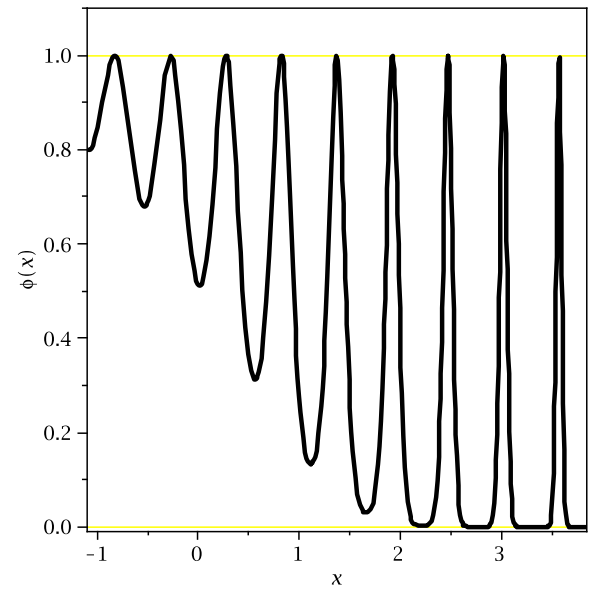

Figure 4: The time evolution of the $p$-adic tachyon field for the special case where the initial function crosses $\phi_{0}\left(x^{+}\right)=1$ at some point on $x^{+} \in[-T, 0]$. In this special case the tachyon undergoes sustained periodic oscillations between the minimum and maximum of the potential. It may be possible to interpret this solution as an array of space-like branes (S-branes). We set $p=3$ and $V^{+}=1$ for illustration. The horizontal yellow lines illustrate the critical values $\phi=0$ and $\phi=1$.

again assume a light-like dilaton profile of the form $\Phi(x)=-V^{+} x^{-}$. The Lagrangian is [27]

$$
\mathcal{L}=\frac{e^{V^{+} x^{-}}}{g_{s}^{2}}\left[-\frac{1}{2}(\partial \phi)^{2}+\frac{1}{2} \phi^{2}-\frac{C^{3}}{3}\left(C^{+\square} \phi\right)^{3}\right]
$$

where $g_{s}$ is the open string coupling, the dimensionless constant 4 is $C=3 \sqrt{3} / 4 \cong$ 1.23 and the scalar field $\phi(x)$, again, corresponds to the open string tachyon. The potential is

$$
g_{s}^{2} e^{-V^{+} x^{-}} V(\phi)=-\frac{1}{2} \phi^{2}+\frac{C^{3}}{3} \phi^{3}
$$

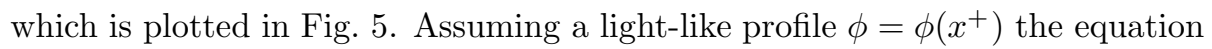
of motion takes the form

$$
\left(V^{+} \partial_{+}-1\right) \phi\left(x^{+}\right)+C^{3} \phi^{2}\left(x^{+}-T\right)=0
$$

where the delay is

$$
T=2 V^{+} \ln C
$$

Equation (21) cannot be solved analytically. However, the method of steps is easy to implement numerically. As in the $p$-adic case, we should subject $\phi_{0}\left(x^{+}\right)$

\footnotetext{
${ }^{4}$ Our $C$ is related to the constant $K$ in employed by Hellerman and Schnabl in [27] as $C=1 / K^{\mathrm{H} . \mathrm{S}}$. and to the constant $K$ employed by Beaujean and Moeller in $[29]$ as $C=K^{\mathrm{B} . \mathrm{M}}$.
} 


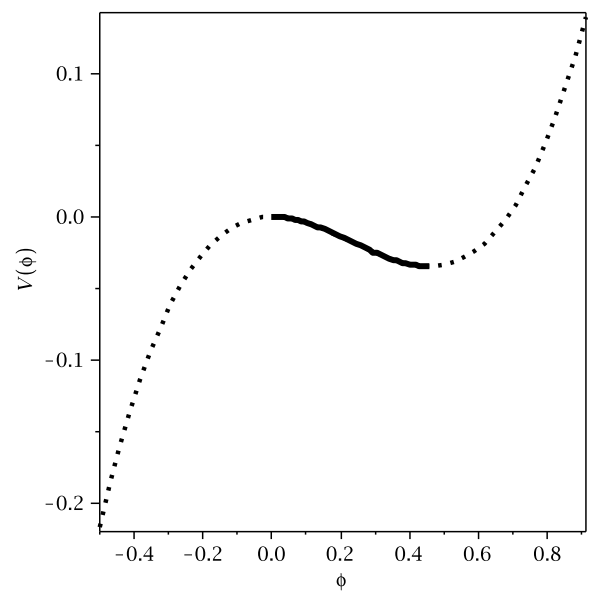

Figure 5: The potential for the SFT tachyon field. The maximum $\phi=0$ correspond to the physical state containing an unstable D-brane while the minimum at $\phi=C^{-3}$ is the "vacuum" containing neither D-brane nor open string excitations. The unbounded region is thought to be associated with the closed string tachyon. The solid part of the potential, $0<\phi<C^{-3}$, denotes the region which can be accessed by physically sensible initial functions.

to some restrictions to ensure that the solutions admit a meaningful physical interpretation in terms of D-brane decay. What class of initial functions are allowed? As in our previous example, the maximum $\phi=0$ corresponds to the physical configuration with an unstable space-filling D-brane while the minimum $\phi=C^{-3}$ corresponds to the empty configuration which contains neither D-brane nor open string excitations. At the minimum, the meaning of the open string variable $\phi$ is obscure. Similarly, solutions rolling down the unbounded $\phi<0$ side of the potential are associated with the closed string tachyon and considered unphysical 39]. All told, the theory (19) admits a robust physical interpretation in terms of D-brane decay only when the initial function satisfies

$$
0<\phi_{0}\left(x^{+}\right)<C^{-3}
$$

which corresponds to the solid region of the potential curve plotted in Fig. 5 . In addition to (23), we should also ensure that the tachyon rolls down the "correct" side of the potential, towards the open string vacuum at $\phi=C^{-3}$. We will discuss this point in more detail shortly, however, a sufficient (but not necessary) condition is the following

$$
\phi_{0}(0)>\phi_{0}(-T)
$$

The condition (24) allows for a variety of non-monotonic behaviours on the interval $x^{+} \in[-T, 0]$, however, it imposes that the "net" motion of the tachyon over this time is to increase. As was the case with (23), the condition (24) is not 
onerous: one expects it to be satisfied for any solution that admits a physical interpretation in terms of D-brane decay.

We have studied the nonlinear solutions obtained using the method of steps and found that the dynamics agree very well with the results of [28] using the diffusion equation approach. As long as the initial function $\phi_{0}\left(x^{+}\right)$satisfies the physical constraints (23)24) then all solutions will eventually settle down to the minimum of the potential in a time comparable to the delay. This behaviour is illustrated in the left panel of Fig. [ 6 for three representative choices of initial function (provided explicitly in Appendix A). It should be emphasized that our numerical evidence for this result is much more exhausitive than the three specific examples plotted in Fig. 6r we have repeated this analysis for a very broad array of initial functions and found qualitatively similar behaviour in all cases.

The Hamiltonian associated with (19) is unbounded below, however, the nonlinear solutions do not display any unstable behaviour as long as (23) is satisfied. Notice also that the late-time dynamics are insensitive to the details of $\phi_{0}\left(x^{+}\right)$.
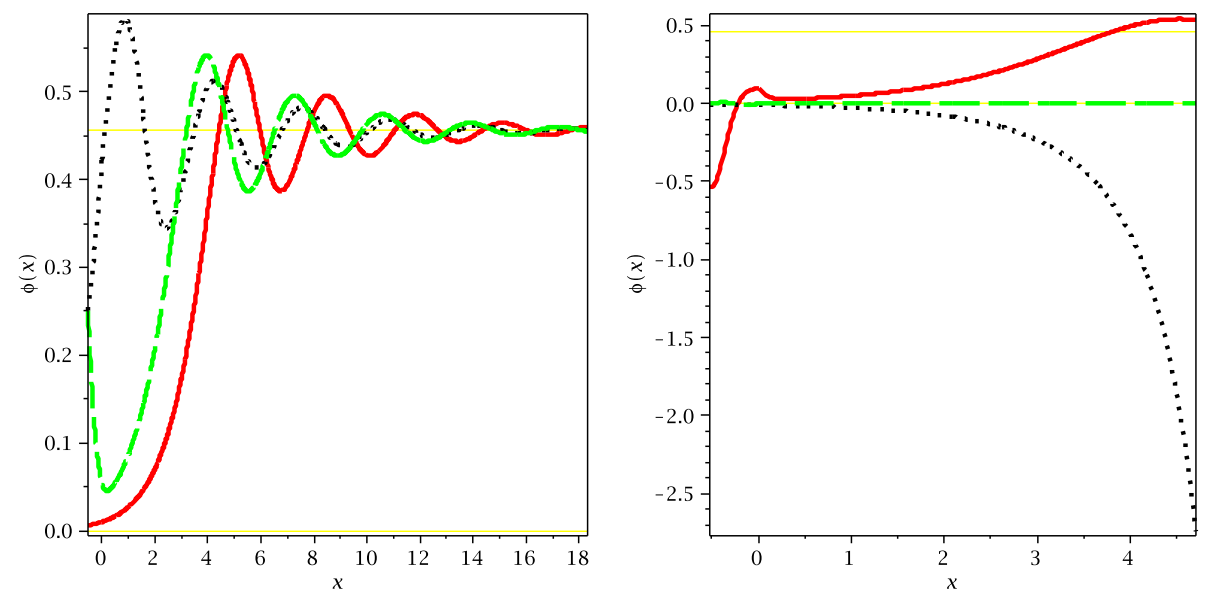

Figure 6: The time evolution of the light-like tachyon field for several representative choices of the initial function. In the left panel we consider only examples which satisfy the constraint $0<\phi_{0}\left(x^{+}\right)<C^{-3}$. At late times the field settles down to the minimum of the potential and the macroscopic $\left(\Delta x^{+} \gg T\right)$ dynamics are largely insensitive to the details of $\phi_{0}\left(x^{+}\right)$. In the right panel we consider three examples where the initial function violates the physical constraint $\phi_{0}\left(x^{+}\right)>0$. The solutions may grow without bound and the late-time dynamics are highly sensitive to the details of $\phi_{0}\left(x^{+}\right)$. In both panels we have set $V^{+}=1$ for illustration and the horizontal yellow lines illustrate the critical values $\phi=0$ and $\phi=C^{-3}$. The explicit choices of $\phi_{0}\left(x^{+}\right)$, for both panels, are provided in Appendix A.

Just like in our last example, the dynamics are very different if we relax the physical constraint (23) on the initial function. If it happens that $\phi_{0}\left(x^{+}\right)<0$ 
on $x^{+} \in[-T, 0]$ then the possibility arises for $\phi\left(x^{+}\right)$to roll down the unbounded part of the potential and grow limitlessly 5 In this case the late-time dynamics will depend very sensitively on the details of $\phi_{0}\left(x^{+}\right)$. This is illustrated in the right panel of Fig. 6 for three representative choices of $\phi_{0}\left(x^{+}\right)$(provided explicitly in Appendix A).

Because we lack an exact analytic solution of (21), it is difficult to prove rigorously that all solutions will relax to the minimum, once (23124) are satisfied. We have provided numerical evidence of this fact. To reinforce our conclusions, we now provide also analytical arguments. Firstly, we note that as soon as $\phi\left(x^{+}\right)$approaches the basin of the closed string vacuum, $\phi=C^{-3}$, then its fate is sealed. All solutions in the vicinity of this point undergo damped oscillations and eventually converge to the minimum, which is easily verified by perturbation theory. Writing

$$
\phi\left(x^{+}\right)=C^{-3}+\delta \phi\left(x^{+}\right)
$$

and linearizing (21) in $\delta \phi$ we obtain

$$
\left[V^{+} \partial_{+}-1+2 C^{-2 V^{+} \partial_{+}}\right] \delta \phi=0
$$

The solutions of (26) take the form

$$
\delta \phi=\sum_{n} a_{n} e^{s_{n} x^{+}}
$$

where $a_{n}$ are arbitrary complex numbers and

$$
V^{+} s_{n}=1+\frac{1}{2 \ln C} W_{n}\left[-\frac{4 \ln C}{C^{2}}\right]
$$

where $W_{n}$ are the branches of the Lambert-W function and $n$ runs over all integer values. One may verify that the $s_{n}$ are complex numbers which appear in conjugate pairs and also that $\operatorname{Re}\left[s_{n}\right]<0$ for all $n$. Thus, all solutions which approach $\phi=C^{-3}$ must eventually converge to the vacuum.

From the preceeding paragraph, we expect that any unbounded solutions must be associated with dynamics near the maximum $\phi=0$. For example, one could imagine starting very close to $\phi=0$ with a large velocity that would allow the tachyon to roll over the maximum and down the unbounded side of the potential. We have claimed that the condition (24) is sufficient to forbid such behaviour (and verified this claim also numerically). Let us now seek to understand better the origin of this constraint. Consider an initial function $\phi_{0}\left(x^{+}\right)$ such that $\phi_{0}(0)$ is very close to zero. Intuitively, such a function represents the most dangerous possibility. By continuity, because $\phi_{0}(0)$ is near the maximum, then so is $\phi_{1}(0)$. By writing the equation of motion (21) in delayed form we can

\footnotetext{
${ }^{5}$ Interestingly, the constraint (23) is sufficient but not necessary to have sensible evolution at late times.
} 
establish the following result:

$$
\begin{aligned}
\left.V^{+} \partial_{+} \phi_{1}\right|_{x^{+}=0} & =\phi_{0}(0)-C^{3} \phi_{0}^{2}(-T) \\
& >\phi_{0}(0)-\phi_{0}(-T)
\end{aligned}
$$

where the inequality on the second line follows from (23). If we further assume (24) then we have

$$
\left.\partial_{+} \phi_{1}\right|_{x^{+}=0}>0
$$

so that the solution on the first know must start near the maximum $\phi=0$ and roll towards the true minimum $\phi=C^{-3}$. We can describe the early stages of this motion using perturbation theory. Writing

$$
\phi=0+\delta \phi
$$

and linearizing (21) we find the growing solution

$$
\delta \phi=a_{0} e^{x^{+} / V^{+}}
$$

The preceeding discussion suggests that our results are rather general. As long as (23|24) are satisfied one expects that all solutions will roll away from the maximum, experiencing exponential growth, until they reach the vicinity of $\phi=C^{-3}$, at which point damped oscillations take over and the solution converges to the minimum. Indeed, precisely this behaviour has been observed in our numerical analysis. This discussion is also consistent with [28].

Finally, let us comment on a "priviledged" class of initial functions. If we wish to minimize derivative singularities (which are presumably unphysical), we should choose $\phi_{0}\left(x^{+}\right)$to be an approximate solution of the equation (21). For solutions rolling away from the maximum, we have a one-parameter family of such functions: $\phi_{0}\left(x^{+}\right)=\epsilon e^{x^{+} / V^{+}}$where $\epsilon \ll 1$ quantifies the initial displacement. With this choice, the condition (24) becomes unnecessary, because the dynamical equation (21) forces solutions near the maximum to grow monotonically. The possibility to roll down the unbounded side of the potential while satisfying (23) arises only for initial functions that are very far from being solutions of (21). Hence, this possibility will typically also be associated with unphysical derivative singularities.

\subsection{Stability and Predictivity}

The dynamics illustrated in Fig. 3 and Fig. 6 are quite remarkable. In both cases the Hamiltonian is unbounded from below. Nevertheless, nonlinear solutions cannot access the unstable directions in phase space starting from an "allowed" initial function $\phi_{0}\left(x^{+}\right)$. The constraint which must be imposed on $\phi_{0}\left(x^{+}\right)$in order to obtain sensible late-time evolution is extremely mild: it amounts to restricting the tachyon to the physically meaningful region of the potential over an interval comparable to the microscopic scale of nonlocality 6

\footnotetext{
${ }^{6}$ In particularly, we do not require that the field sits at an unstable maximum for an infinite amount of time. That would be incompatible with quantum mechanics and, presumably, also with macroscopic causality/predictivity.
} 
These mild physical restrictions on $\phi_{0}\left(x^{+}\right)$have the effect of constraining the theory to a stable subset of its solution space. That this might be possible has been suggested previously, for example by Woodard and Eliezer in [4]. The approach employed here has the advantage of being completely nonperturbative and also physically well-motivated. Moreover, our solutions depend on infinitely many free coefficients and, in this sense, retain information about the full nonlocal structure of the model. (Hence, our approach is different from perturbative localization.)

Suppose that we had adopted a different view of the initial value problem: fixing infinitely many derivatives $\partial_{t}^{(n)} \phi$ at $t=0$, instead of the initial function formulation (6). In this picture we would have uncovered an "island of stability" in initial condition space which leads to stable late-time dynamics. Constraining the theory to the island of stability requires a seemingly delicate and contrived tuning amongst the infinite tower of initial conditions. However, this tuning seems contrived only because one is using a highly unnatural formulation of the initial value problem. In light of the initial function formulation (6) we see that, in fact, the constraints imposed by late-time stability are very mild.

What becomes of predictivity in our delayed models (9) and (21)? As discussed in section 2, there is a breakdown of predictivity on microscopic scales, $\Delta x^{+} \ll T \sim V^{+}$. In essence, this occurs because our ignorance about the past history of the system makes the initial function $\phi_{0}\left(x^{+}\right)$inherently ambiguous. However, as we have seen, the late-time dynamics are largely insensitive to $\phi_{0}\left(x^{+}\right)$, provided it belongs to the class of allowed initial functions. Hence, we are still able to recover predictivity on macroscopic scales, $\Delta x^{+} \gg T$.

We view the breakdown of microscopic predictivity as a necessary feature of working with a fundamentally nonlocal theory. Indeed, it seems quite natural: we should not expect to be able to unambiguously resolve dynamics on times comparable to the underlying scale of nonlocality.

Obviously, our conclusions about the nonperturbative stability of equations (9) and (21) are purely classical. One might worry that instabilities re-appear at the loop level (see [37] for a related discussion) or that the tachyon field $\phi$ can tunnel to the unbounded region of the potential, violating the physical restrictions (11) and (23). We note, however, that in string theory the situation may be rather subtle. It is not clear if the physical degree of freedom associated with the tachyon field even exists on the unbounded region of the potential, or at the local minimum 7

To summarize: the string theory models (9) and (21) are nonperturbatively stable and make unambiguous predictions over time scales large compared to the

\footnotetext{
${ }^{7}$ At the local minimum the D-brane has disappeared and there can be no open string degrees of freedom. It may be that $\phi$ somehow encodes the closed string gas which is the outcome of the brane decay [41] or it may simply be that the $\phi$ disappears from the theory. The latter interpretation is similar to what happens to the inflaton field during brane/anti-brane inflation [11]. At the end of inflation, the mobile brane annihilates with its anti-brane and the inflaton field (corresponding to the inter-brane separation) no longer exists as a physical state in the theory.
} 
delay, provided $\phi_{0}(t)$ belongs to a class of allowed initial function. Obviously, our optimistic conclusions have relied heavily on the delayed form of the prototype model (2) and the formulation (6) of the initial value problem. Only a very privileged class of nonlocal theories (those having compact support [4]) admit such a formulation. One might wonder how much of our analysis can be carried over to a more realistic QFT framework. We will investigate this question in section 4 .

\section{The Initial Function Formulation Applied to Generic Nonlocal Field Theory Models}

Our goal now is to determine the extent to which the optimistic conclusions of section 3 carry over to a general nonlocal QFT framework. To this end, we first introduce the very general class of nonlocal field theories. Next, we will show how the simple-minded inclusion of dissipation into the dynamics leads to delay-type nonlocality and an initial value problem of the form (6).

\subsection{Prototype Nonlocal Field Theory}

Consider the prototype nonlocal field theory

$$
\mathcal{L}=\frac{1}{2} \phi F(\square) \phi-V_{\mathrm{int}}(\phi), \quad V_{\mathrm{int}}(\phi)=\frac{g}{3 !} \phi^{3}+\frac{\lambda}{4 !} \phi^{4}+\cdots
$$

This model subsumes known examples from SFT [1], $p$-adic string theory [6] and also cosmology [16, 18, 17, 21, 22, 23]. The full scalar potential is

$$
\begin{aligned}
V(\phi) & =-\left.\mathcal{L}\right|_{\square=0} \\
& =-\frac{F(0)}{2} \phi^{2}+V_{\mathrm{int}}(\phi) \\
& \equiv \frac{m^{2}}{2 !} \phi^{2}+\frac{g}{3 !} \phi^{3}+\frac{\lambda}{4 !} \phi^{4}+\cdots
\end{aligned}
$$

with $m^{2} \equiv-F(0)$.

The nonlocal structure of (34) is encoded in the pseudo-differential operator $F(\square)$. In most cases of interest this can be represented as a convergent series expansion

$$
F(\square)=\sum_{n=0}^{\infty} f_{n} \square^{n}
$$

where the power $\square^{n}$ is to be understood as $n$-fold composition of the d'Alembertian operator with itself. By assumption the generatrix $F(z)$ is therefore an analytic function of the complex variable $z$. The Weierstrass factorization theorem ensures that we can write

$$
F(z)=\Gamma(z) \prod_{a=1}^{M}\left(z-m_{a}^{2}\right)^{r_{a}}
$$


with $\Gamma(z)$ having neither zeros nor poles at finite $z$. The propagator $G\left(p^{2}\right) \sim$ $1 / F\left(-p^{2}\right)$ therefore has precisely $M$ poles at the locations $p^{2}=-m_{a}^{2}$, with the $a$-th pole being of integer order $r_{a}$.

The field equation derived from (34) takes the form

$$
F(\square) \phi=V_{\text {int }}^{\prime}(\phi)
$$

The initial value problem associated with (38) has been studied in 24, 31] using the formal generatrix calculus, and also in [4, 18, 32, 33, 34, 32, 35] using alternative approaches.

\subsection{Multi-Particle Form of the Action}

Let us now re-write the prototype Lagrangian (34) in a form which is equivalent but which makes certain key features more transparent. (See also [25, 12].)

We assume a kinetic function of the form (37) and perform the field redefinition 8

$$
\varphi=\Gamma(\square)^{1 / 2} \phi
$$

so that the Lagrangian (34) becomes

$$
\mathcal{L}=\frac{1}{2} \varphi \prod_{a=1}^{M}\left(\square-m_{a}^{2}\right)^{r_{a}} \varphi-V_{\text {int }}\left[\Gamma^{-1 / 2}(\square) \varphi\right]
$$

Next, we introduce $M$ independent "constituent" fields defined as

$$
\varphi^{a}=\prod_{b \neq a}\left(\square-m_{b}^{2}\right)^{r_{b}} \varphi
$$

The "collective" field variable $\phi$ of the original theory is a superposition of these constituents:

$$
\phi=\Gamma^{-1 / 2}(\square) \sum_{a=1}^{M} \eta_{a} \varphi^{a}
$$

where the coefficients $\eta_{a}$ define a partial fraction decomposition of the quantity $H(z) \equiv F(z) / \Gamma(z)$ as

$$
\sum_{a=1}^{M} \frac{\eta_{a}}{\left(z-m_{a}^{2}\right)^{r_{a}}}=\left[\prod_{a=1}^{M}\left(z-m_{a}^{2}\right)^{r_{a}}\right]^{-1}
$$

Now, the Lagrangian (34) can be written in the decoupled form

$$
\mathcal{L}=\sum_{a=1}^{M} \frac{1}{2} \eta_{a} \varphi^{a}\left(\square-m_{a}^{2}\right)^{r_{a}} \varphi^{a}-V_{\mathrm{int}}\left[\Gamma^{-1 / 2}(\square) \sum_{b=1}^{M} \eta_{b} \varphi^{b}\right]
$$

\footnotetext{
${ }^{8}$ This is an allowed manipulation because $\Gamma(z)$ contains neither zeros nor poles.
} 
Equation (44) is the main result of this sub-section, it shows how the Lagrangian (34) for the "collective" field $\phi$ can be decomposed into a theory of $M$ "constituent" fields $\varphi^{a}$ (one for each pole of the propagator). Notice that we have not made any particular assumptions about the geometry of the background spacetime, nor have we employed perturbation theory. In Appendix B we show how to use the multi-particle form of the action (44) to construct the perturbative Hamiltonian and look for constituent ghost excitations.

Using (41), (42) and (43) is is possible to re-write the dynamical equation (38) in the form

$$
\left(\square-m_{a}^{2}\right)^{r_{a}} \varphi_{a}=\Gamma^{-1 / 2}(\square) V_{\text {int }}^{\prime}\left[\Gamma^{-1 / 2}(\square) \sum_{b} \eta_{b} \varphi^{b}\right]
$$

(Notice that here $V_{\text {int }}^{\prime}$ is the derivative of $V_{\text {int }}$ with respect to the collective field $\phi$, and not with respect to the constituent $\varphi^{a}$.)

\subsection{Dissipation and Delays}

Our examples in section 3 both inherit their delayed structure from some source of dissipation in the dynamics. We can model the effects of dissipation on our prototype nonlocal QFT (34) is a very simple way, by adding a friction term to the d'Alembertian. Thus, we take

$$
\square=-\partial_{t}^{2}-\beta \partial_{t}
$$

when acting on a homogeneous function. There are many ways to motivate the appearance such a friction term. Obviously, we could obtain (46) by working in an expanding FRW universe. In this case we would have $\beta=3 H$ where $H=\dot{a} / a$ is the Hubble scale. More generally, one might imagine motivating the appearance of such a term by supposing that the motion of $\phi$ leads to particle production, in which case $\beta$ would be related to the imaginary part of the effective action. (See, for example, [48].)

To see how the simple-minded modelling of dissipation encapsulated in equation (46) can lead to delay-type nonlocality, let us assume a kinetic function of the form (37) with

$$
\Gamma(\square)=e^{-\alpha \square}
$$

This type of nonlocality is ubiquitous in string theory; factors of the form $e^{-\alpha \square}$ typically arise at the vertices of Feynman diagrams. Using (46) and (47) we can re-write the constituent equation of motion (45) in the form

$$
\left(-\partial_{t}^{2}-\beta \partial_{t}-m_{a}^{2}\right)^{r_{a}} \varphi^{a}(t)=e^{-\frac{\alpha}{2} \partial_{t}^{2}} V_{\mathrm{int}}^{\prime}\left[e^{-\frac{\alpha}{2} \partial_{t}^{2}} \sum_{b} \eta_{b} \varphi^{b}(t-\alpha \beta)\right]
$$

where we assume that $\beta$ is a constant. We now introduce yet another set of basis field $\$$

$$
\psi^{a}(t) \equiv e^{-\frac{\alpha}{2} \partial_{t}^{2}} \varphi^{a}(t)
$$

\footnotetext{
${ }^{9}$ Once again, this manipulation is allowed because $e^{-\alpha s^{2} / 2}$ has neither zeroes nor poles.
} 
Finally, the dynamical equation (48) for the constituents takes the form

$$
\left(-\partial_{t}^{2}-\beta \partial_{t}-m_{a}^{2}\right)^{r_{a}} \psi^{a}(t)=e^{-\alpha \partial_{t}^{2}} V_{\text {int }}^{\prime}\left[\sum_{b} \eta_{b} \psi^{b}(t-T)\right]
$$

where the delay is

$$
T=\alpha \beta
$$

This we suppose is positive. Equation (50) is the main result of this sub-section.

Equation (50) is almost exactly of the form (2). The only complication is the nonlocal operator $e^{-\alpha \partial_{t}^{2}}$ on the right hand side. We can seek solutions by the method of steps. (It might be that this approach does not access the full solution space.) Each constituent $\psi^{a}(t)$ is split into solution segments $\psi^{a}(t)=\psi_{i}^{a}(t)$ on the interval $t \in[(i-1) T, i T]$ with integer $i$. (Here the upper index on the field denotes the constituent label while the lower index labels the segment.) The $i$-th solution segment can be obtained recursively as

$$
\left(-\partial_{t}^{2}-\beta \partial_{t}-m_{a}^{2}\right)^{r_{a}} \psi_{i}^{a}(t)=e^{-\alpha \partial_{t}^{2}} V_{\mathrm{int}}^{\prime}\left[\sum_{b} \eta_{b} \psi_{i-1}^{b}(t-T)\right]
$$

On the first segment we have an arbitrary initial function for each constituent field

$$
\psi^{a}(t)=\psi_{0}^{a}(t) \quad \text { on } \quad t \in[-T, 0]
$$

Since equation (52) is $2 r_{a}$-th in time derivatives, it follows that we can make $\psi^{a}(t)$ and its first $2 r_{a}-1$ derivatives continuous at the knots $t=0, T, 2 T, \cdots$

It is worth pausing to admire the simplicity of equation (52). We can construct solutions of a nonlinear differential equation of infinite order simply by solving a set of $M$ inhomogeneous linear equations, each of which is finite order in derivatives. The only complication is the appearance of the nonlocal operator $e^{-\alpha \partial_{t}^{2}}$ in the source term.

In this section we have shown that the naive inclusion of dissipation (46) into the general prototype nonlocal theory (34) leads to delay-type nonlocality, as manifested in the dynamical equation (50). Even though the nonlocality is not purely of the delay type, we are still able to employ the method of steps. In the next section we will show how this approach can be used to construct efficient and numerically stable time-dependent solutions.

Equation (53) provides a novel new way of thinking about the initial value problem for the nonlocal theory (34). Each pole of the propagator contributes a "constituent" field $\varphi^{a}$ with its own independent initial function.

Of course, the fact that a delayed formulation (52) with initial value problem (53) is possible does not imply that the theory (34) is stable or usefully predictive in any sense. These key questions will depend sensitively on the underlying field theory and the class of allowed initial functions. It seems necessary to proceed on a case-by-case basis. In the next section, we will consider a specific example. 


\section{A Case Study: Cosmological D-Brane Decay}

\subsection{Delayed Formulation and the Method of Steps}

In the last section we showed how the simple-minded inclusion of dissipation leads to a delayed formulation of the dynamics in a very general nonlocal QFT context. Here we consider a specific example: D-brane decay in a background de Sitter space-time. This toy model may be relevant for nonlocal models of the early universe [21, 22, 23] and also quintessence [16]. We employ the same level zero truncation of SFT as was studied in subsection 3.2 however, this time we take a constant dilaton profile and minimally couple $\phi$ to Einstein gravity:

$$
\mathcal{L}=\frac{1}{g_{s}^{2}}\left[\frac{1}{2} \phi C^{-2 \square}(\square+1) \phi-\frac{C^{3}}{3} \phi^{3}\right]
$$

(see [5] for more details). This example is consistent with our prototype model (34) and also the more restrictive class of theories considered in subsection 4.3 . The equation of motion

$$
C^{-2 \square}(\square+1) \phi=C^{3} \phi^{2}
$$

admits infinitely many initial conditions and the Hamiltonian associated with (54) is unbounded below [24] (see Appendix C for a review). Our goal is to derive a delayed formulation of this equation and argue that the theory can be constrained to a stable subset of its solution space at the expense of certain mild physical restrictions on the class of allowed initial functions.

In a background de Sitter space-time the equation of motion (55) can be written as

$$
\left(\partial_{t}^{2}+3 H \partial_{t}-1\right) \phi(t)=-C^{3-2 \partial_{t}^{2}} \phi^{2}(t-T), \quad T \equiv 6 H \ln C
$$

Following subsection 4.3 we can employ the method of steps. The $i$-th solution segment $\phi_{i}(t)$ is constructed recursively as

$$
\left(\partial_{t}^{2}+3 H \partial_{t}-1\right) \phi_{i}(t)=-C^{3-2 \partial_{t}^{2}} \phi_{i-1}^{2}(t-T)
$$

On the first segment $(i=0)$ we have the initial function: $\phi(t)=\phi_{0}(t)$ on $t \in[-T, 0]$. Since the differential operator on the left hand side of (57) is second order, we can demand that the field and it's first derivative are both continuous at the points $t=i T$ where the solution segments are joined.

\subsection{Allowed Initial Functions}

Which restrictions on $\phi_{0}(t)$ are necessary to constraint the theory (56) to a stable subset of its solution space? Following the analysis of subsection 3.2 we might hope that the simple physical requirement $0<\phi_{0}(t)<C^{-3}$ will be sufficient. However, this is not the case, we will also need to bound the derivatives of $\phi_{0}(t)$.

We can see which class of $\phi_{0}(t)$ will lead to late-time instability in a very simple way, by inspection of the delayed formulation (57). For a given initial 
function $\phi_{0}(t)$ we can expand the quantity $-C^{3} \phi_{0}^{2}(t-T)$ on the interval $-T \leq$ $t \leq 0$ as

$$
-C^{3} \phi_{0}^{2}(t-T)=\sum_{n} \alpha_{n} e^{2 \pi i n t / T}
$$

(For the sake of this argument the reader may interpret the summation over $n$ as either discrete or continuous.) Now consider the $i=1$ solution segment, $\phi_{1}(t)$. This obeys an inhomogeneous equation

$$
\left(\partial_{t}^{2}+3 H \partial_{t}-1\right) \phi_{1}(t)=\sum_{n} C^{8 \pi^{2} n^{2} / T^{2}} \alpha_{n} e^{2 \pi i n t / T}
$$

Then the dominant contribution to the source term obviously comes from the mode $n$ which maximizes the quantity $C^{8 \pi^{2} n^{2} / T^{2}} \alpha_{n}$. Unless the coefficients in (58) tend to zero sufficiently fast, then it may happen that the dominant contribution to the source comes from some high frequency mode with $n \gg 1$. If that happens then the particular solution of (57) will go like $\phi_{1}(t) \sim e^{i \omega t}$ with $\omega \gg H$. Carrying through the same procedure, the next segment oscillates even faster, as $\phi_{2}(t) \sim e^{2 i \omega t}$. And so on. At each successive step the energy cascades into higher-and-higher frequencies so that the solution will oscillate ever more rapidly. This is precisely the usual Ostrogradski instability. It is clear that in order to evade this pathological behaviour we must ensure that the Fourier coefficients $\alpha_{n}$ tend to zero sufficiently quickly at high frequency.

Establishing a necessary condition on $\phi_{0}(t)$ for late-time nonperturbative stability is very difficult. Therefore we will instead seek a sufficient condition. An obvious candidate is the constraint $0<\phi_{0}(t)<C^{-3}$ and also that

$\partial_{t}^{(n)} \phi_{0}(t) / \phi_{0}(t) \lesssim H^{n}$. In the next section we will test this conjecture using fully nonlinear numerical simulations. Although this constraint is slightly stronger than those which were considered in section 3 , it is still far from onerous. Indeed, we might imagine that this type of consistency condition could be established dynamically within the context of a more realistic model of dissipation.

\subsection{Numerical Methods and Nonlinear Solutions}

We now implement numerically the method of steps (57). Obviously, to proceed we need to a faithful numerical implementation of the infinite order operator $C^{-2 \partial_{t}^{2}}$. We could proceed by decomposing $\phi_{i-1}^{2}(t-T)$ into a Fourier series on the segment $t \in[(i-1) T, i T]$. Then each member of the sum is an eigenfunction of $\partial_{t}^{2}$ so that $C^{-2 \partial_{t}^{2}}$ acts in a simple way, term-by-term. However, this idea turns out to be a very bad approach. The problem is that the Gibbs phenomenon introduces errors at the points $t=i T$ where the solution segments are joined. These errors propagate and become very large after just a few steps. A much better approach is to represent the quantity $\phi_{i-1}^{2}(t-T)$ using an expansion in Chebyshev polynomials. When this expansion is truncated to order $2 N$ (with $N$ integer) then the pseudo-differential operator $C^{-2 \partial_{t}^{2}}$ is equivalent to the partial sum $\sum_{n=0}^{N} \frac{(-2 \ln C)^{n}}{n !} \partial_{t}^{(2 n)}$ and is straightforward to implement numerically. 
When the dissipation is sufficient to allow the field to settle down to the minimum at late times, then our iterative solution of (56) is very efficient and numerically stable, a significant improvement over higher order perturbation theory or the diffusion equation formulation (of course, our method is also considerably less general than those alternative approaches). These results are robust when the number of steps or the order of the Chebyshev polynomials is increased. On the other hand, when the dissipation is small then the method breaks down within just a few steps. However, since we regard wildly unstable solutions as unphysical, this is not a serious drawback.

We have investigated numerically the sufficient conditions on $\phi_{0}(t)$ for latetime stability. The results confirm our previous intuition. We will have $\phi \rightarrow C^{-3}$ as $t \rightarrow \infty$ as long as $0<\phi_{0}(t)<C^{-3}$ on $t \in[-T, 0]$ and the derivatives of $\phi_{0}(t)$ are not too large as compare to the coefficient of dissipation 10 These dynamics are illustrated in Fig. 7.

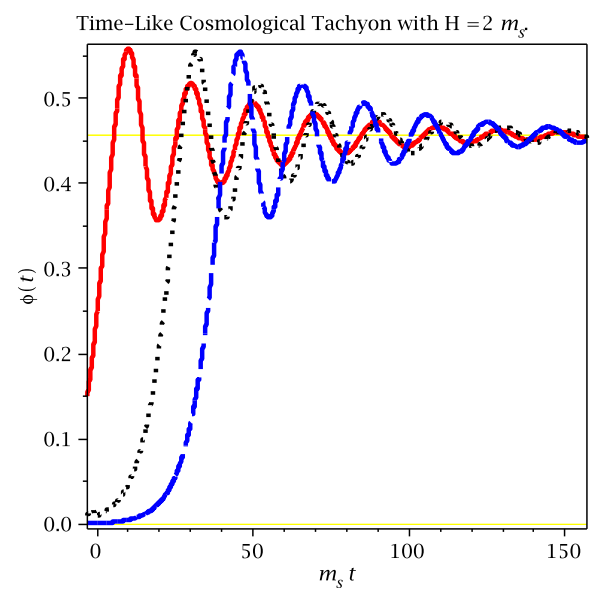

Figure 7: The time-evolution of the time-like SFT tachyon in the presence of cosmological (de Sitter) friction for several representative choice of initial function, $\phi_{0}(t)$. We set $H=2 m_{s}$ for illustration. Provided the constraint $0<\phi_{0}(t)<C^{-3}$ is satisfied, and also that the derivatives of $\phi_{0}(t)$ are not too large, then the field will eventually settle down to the true minimum at late times. The horizontal yellow lines illustrate the critical points $\phi=0$ and $\phi=C^{-3}$. The explicit choices of $\phi_{0}\left(x^{+}\right)$are provided in Appendix A.

Our results suggest that nearly any source of dissipation will allow us to salvage sensible dynamics from the theory (54), even without making restrictive assumptions about the symmetries of the solution. We find this prospect encouraging since the rolling tachyon is supposed to be decaying into closed string excitations [40] which were not taken into account in the effective de-

\footnotetext{
${ }^{10}$ Quantitatively we can demand that the Laplace transform of $\phi_{0}(t)$ is analytic inside a circular contour of radius $H$. We stress that this is a sufficient, but not necessary, constraint.
} 
scription (54). Thus, our results may provide a plausible mechanism by which the consistent dynamical inclusion of closed strings could allow SFT to evade the Ostrogradski instability. At the more practical level, this general prescription helps to establish the viability of nonlocal cosmological models.

\subsection{Wild Oscillations and Allowed Initial Functions}

It has been argued previously that the dynamics of tachyon condensation are stable for the special case where $\phi(t) \rightarrow 0$ as $t \rightarrow-\infty$. For example, this was discussed in [29] for the light-like dilaton models discussed in section 3 (although in that paper the analysis went beyond our simple level-0 truncation). See also 32. It is not clear if demanding that the field sit at an unstable maximum can be consistent with quantum mechanics (or, for that matter, macroscopic predictivity). It should therefore be emphasized that our class of "allowed" initial functions is much broader than this very special subset of solution space. We only require certain qualitative restrictions on the dynamics of $\phi(t)$ over a microscopic interval $\Delta t \sim m_{s}^{-1}$. Moreover, our solutions depend on infinitely

many free parameters and, in this sense, encode information about the full nonlocal structure of the equations. Nevertheless, it is worth comparing our analysis with the special case where $\phi(t) \rightarrow 0$ as $t \rightarrow-\infty$. Because $\phi=0$ is a surface of degeneracy, there is only a single growing mode which can be accessed by naive perturbation theory about the unstable maximum: $\phi_{0}(t)=\epsilon C^{-3} e^{r+t}$, with $\epsilon \ll 1$ and $r_{+}=-\frac{3 H}{2}+\frac{1}{2} \sqrt{9 H^{2}+4}$. Using the method of steps, we have confirmed numerically that this special class of initial function always leads to sensible late-time evolution, at least when $H \gtrsim 1$. Notice that this special function is not the unique perturbative solution of equation (56) which satisfies $\phi(t) \rightarrow 0$ as $t \rightarrow-\infty$. Using the technique described in the appendix of [28] we could easily constuct a family of such solutions which depend on infinitely many free parameters.

In passing, it is worth noting that the wild oscillations of rolling tachyon solutions, which are a symptom of the Ostrogradsi instability, are not always viewed as pathological. This comes down to a delicate issue of interpretation. It is nontrivial to relate $\phi$ to physical observables in SFT. Perhaps the ghostly constituent excitations are pure gauge. In [26] it was argued that the unstable dynamics of $\phi$ can be interpreted as describing the closed string physics associated with Sen's tachyon matter [49]. It is not clear to us how (or if) this claim addresses the issue of predictivity discussed in the introduction. (See also [50, 51, 52] for related discussions.) In this work our aim is to address stability and predictivity in a general QFT context, without relying on intrinsically stringy physics. Hence we regard any wildly unstable solutions as unphysical.

\section{Conclusions}

In this work we proposed a new formulation of the initial value problem for nonlocal theories with infinitely many derivatives. This new approach is less general than the pseudo-differential operator technique advanced in [24, 31], 
however, it has considerable conceptual appeal. In particular, the underlying nonlocality of the model is manifest; the Cauchy surface has been "smeared out" over a finite width.

We have found that this mathematical re-formulation leads to a novel new perspective on the key problems of stability and predictivity which plague nonlocal models. By imposing mild physical restrictions on $\phi_{0}(t)$, it is possible to constraint the theory to a stable subset of its solution space. Moreover, the constrained theory makes robust predictions for dynamics on macroscopic scales. Although our claims are limited only to a small number of specific examples, we expect that similar results might be possible more generally. It is our view that these restrictions on the initial function should be viewed as a part of the definition of the theory.

Philosophically, our prescription is very similar to approaches which have been advocated previously for higher derivative theories of finite order. Both the Cutkowski et al. contour deformation [43, 44, 45] and also the Hertog and Hawking [46] approach are, classically, equivalent to adopting stability as a future boundary condition. Both these approaches lead to a violation of microscopic causality, however, it is thought that macroscopic causality survives [46, 47]. In the initial function formulation we have an elegant and physically well-motivated way to impose late-time stability for models with infinitely many derivatives. Our approach is nonperturbative and retains information about the full nonlocal structure of the theory. Notice that microscopic causality is manifestly violated in this formulation of the initial value problem. We view this, along with the breakdown of microscopic predictivity, as a necessary feature of working with a fundamentally nonlocal theory. In fact, these features seem rather natural: we should not expect to be able to unambiguously resolve dynamics on times comparable to the underlying scale of nonlocality.

It is not our goal to claim that nonlocal models are generically sensible or phenomenologically viable. Rather, we believe that fundamental nonlocality is ubiquitous in well-motivated microscopic models and, therefore, it behooves us to try to understand such constructions from a general perspective. By studying the connection between dissipation and delays, we have arrived at a new formulation of the initial value problem and a novel new perspective on the key issues of stability and predictivity. These general lessons may be applied in a variety of contexts. For example, the rolling open string tachyon is, physically, supposed to be decaying into closed string excitations [40] which are usually not taken into account in SFT. Plausibly, the dissipation induced by this type of particle production has a dynamical effect which is similar to the dilaton gradient in section 3 or the cosmological friction in section 5. Thus, we have elucidated a mechanism by which the consistent inclusion of closed strings might enable SFT to escape the Ostrogradski instability. A strength of our approach is that we do not rely on any intrinsically stringy features of the underlying model. Thus, our results may readily be applied to more general nonlocal QFT models (in particular, to nonlocal cosmological models).

There are a number of directions for future work on this subject. Obviously, our results for nonlinear stability have been rather specific and model- 
dependent. It would be very interesting to understand the connection between dissipation and delays in more general models, for example where the coefficient of dissipation is time-dependent (as would arise in a self-consistent cosmology).

Our analysis has been entirely classical, which is an obvious limitation. Instabilities might re-appear at the loop level, or it might be that the field can tunnel quantum mechanically to some unstable configuration even when the unstable directions in phase space are not classically accessible from an allowed initial state (perhaps similarly to [53]). At the current level of understanding, it seems that perturbation theory is best suited to study quantization while the method of steps is best suited to study nonperturbative dynamics in the classical regime. However, it might be possible to incorporate quantization directly into

the delayed formulation. One could imagine imposing $\left[\phi_{0}(t, \mathbf{x}), \dot{\phi}_{0}(t, \mathbf{x})\right] \neq 0$ on the initial segment $-T \leq t \leq 0$ and then using the method of steps to construct the q-number field $\phi(t, \mathbf{x})$ on $t>0$. It would be interesting to understand how the imposition of physical constraints on $\phi_{0}$ can be performed in a quantum theory.

One might also imagine trying to compute statistical properties of the $t>0$ dynamics by performing a functional average of the solution over allowed $\phi_{0}(t)$. We find this idea fascinating, however, it is not clear how to put a measure on the space of initial functions. We have investigated this idea using the exact solution (12) where the averaging can be performed analytically. In this particular case it seems that the ambiguity in choosing the averaging procedure is not less sever than the ambiguity in choosing a realization of the initial function.

It is interesting to note that our physical restrictions on $\phi_{0}(t)$ are not so stringent that they forbid violations of the Null Energy Condition (NEC). In [28] this was shown explicitly for the toy $p$-adic model studied in section 3 . Violations of the NEC play a key role in cyclic/bouncing cosmologies, such as the new ekpyrotic model [54], but are notoriously difficult to obtain in a consistent microscopic framework. It is possible that our formalism could be used to construct sensible, NEC-violating nonlocal field theories. We leave this, and other interesting possibilities, to future investigations.

\section{Acknowledgments}

I am grateful to a number people for helpful discussions, correspondence and collaboration over the last few years which helped to shape the ideas in this text, including T. Biswas, J. Cline, N. Kamran, N. Moeller, S. Patil, I. Sachs, R. Woodard and especially D. Mulryne and N. Nunes.

\section{APPENDIX A: Initial Functions for Numerical Analysis}

In this appendix we summarize the explicit initial functions used to generate the numerical results Fig. 6 and Fig. 7. In the left panel of Fig. 6 the solid red, 
dotted black and dashed green curves correspond, respectively, to the functions:

$$
\begin{aligned}
& \phi_{0}\left(x^{+}\right)=10^{-2} e^{x^{+}} \\
& \phi_{0}\left(x^{+}\right)=0.25 e^{x^{+}+T} \\
& \phi_{0}\left(x^{+}\right)=0.25 e^{-3\left(x^{+}+T\right)}
\end{aligned}
$$

In the right panel of Fig. 6 the solid red, dotted black and dashed green curves correspond, respectively, to the functions:

$$
\begin{aligned}
& \phi_{0}\left(x^{+}\right)=0.1\left[1-10 e^{-25\left(x^{+}+T\right)^{2}}\right] e^{x^{+}} \\
& \phi_{0}\left(x^{+}\right)=-10^{-2} e^{x^{+}} \\
& \phi_{0}\left(x^{+}\right)=10^{-2} \sin \left[\frac{2 \pi x^{+}}{T}\right] e^{x^{+}}
\end{aligned}
$$

In Fig. 7 the solid red, dotted black and dashed green curves correspond, respectively, to the functions:

$$
\begin{aligned}
& \phi_{0}(t)=0.25 e^{+r_{+} t} \\
& \phi_{0}(t)=10^{-2}\left[1+0.85 t^{2} \cos (t / 2) e^{t / 2}\right] \\
& \phi_{0}(t)=10^{-3} e^{r_{+} t}
\end{aligned}
$$

where $r_{+}=-\frac{3 H}{2}+\frac{1}{2} \sqrt{9 H^{2}+4}$, as defined in subsection 5.4

\section{APPENDIX B: Conditions for a Bounded Hamiltonian}

In general the interactions in (44) will be nonlocal and the dynamics are very complicated. We can gain some insight into the dynamics of the theory by adopting a perturbative approach. We write the each constituent field as

$$
\varphi^{a}=\delta \varphi^{a}
$$

and expand the Lagrangian (44) order-by-order in perturbation theory

$$
\mathcal{L}=\delta \mathcal{L}^{(2)}+\delta \mathcal{L}^{(3)}+\cdots
$$

where the quadratic part $\delta \mathcal{L}^{(2)}$ gives the linearized equations of motion and the higher order contributions encode the effects of interactions. The quadratic Lagrangian decomposes into constituents as

$$
\delta \mathcal{L}^{(2)}=\sum_{a=1}^{M} \delta \mathcal{L}_{a}^{(2)}, \quad \delta \mathcal{L}_{a}^{(2)}=\frac{\eta_{a}}{2} \delta \varphi^{a}\left(\square-m_{a}^{2}\right)^{r_{a}} \delta \varphi^{a}
$$

Obviously the quadratic part of the Hamiltonian must also admit a similar decomposition

$$
\delta \mathcal{H}^{(2)}=\sum_{a=1}^{M} \delta \mathcal{H}_{a}^{(2)}
$$


where each $\delta \mathcal{H}_{a}^{(2)}$ is constructed from $\delta \mathcal{L}_{a}^{(2)}$.

Under what conditions can the perturbative Hamiltonian (B-4) Hamiltonian be bounded below? If $r_{a}>1$ for any $a$ then $\delta \mathcal{L}_{a}^{(2)}$ must depend non-degenerately on time derivatives higher than than first order. By the Ostrogradski theorem [33], it follows that $\delta \mathcal{H}_{a}^{(2)}$ must be unbounded below. Hence, only theories with $r_{a}=1$ for all $a$ yield a bounded Hamiltonian. In this case the coefficient $\eta_{a}$ can be computed easily from the residues of the propagator at the pole $m_{a}^{2}$ :

$$
\eta_{a}=\oint_{C_{a}} \frac{d z}{2 \pi i} \frac{1}{H(z)}=\frac{1}{H^{\prime}\left(m_{a}^{2}\right)} \quad \text { where } \quad H(z) \equiv \frac{F(z)}{\Gamma(z)}=\prod_{a=1}^{M}\left(z-m_{a}^{2}\right)
$$

(The contour $C_{a}$ only encloses the pole at $z=m_{a}^{2}$.) If any $\eta_{a}$ are negative, then (44) must contain ghosts. If $M>1$ then, because $H(z)$ is analytic, the residues must flip sign and some $\mathcal{H}_{a}$ will be unbounded below. Therefore the only possibility to construct a perturbatively ghost-free theory is when the propagator $G\left(p^{2}\right) \sim 1 / F\left(-p^{2}\right)$ contains at most a single pole.

We have implicitly assumed that $m_{a}^{2}$ is real valued for all $a$. The case of complex mass-squared is slightly more subtle since now the $\eta_{a}$ coefficients are complex, as are the fields $\delta \varphi^{a}$. Hermiticity of the action requires that such complex poles arise in conjugate pairs, so that if $m^{2}$ is a zero of $F(z)$ than so must be $\left(m^{2}\right)^{\star}$. Let us suppose that $m_{1}^{2}$ and $m_{2}^{2}$ are complex conjugate pairs. Then, reality of the solution implies that $\delta \varphi^{2}=\left(\delta \varphi^{1}\right)^{\star}$ and the relevant part of the Lagrangian is

$$
\frac{\eta_{1}}{2} \delta \varphi^{1}\left(\square-m_{1}^{2}\right) \delta \varphi^{1}+\text { c.c. }=-\frac{1}{2} K_{A B} \partial_{\mu} \delta \psi^{A} \partial^{\mu} \delta \psi^{B}-\frac{1}{2} M_{A B} \delta \psi^{A} \delta \psi^{B}
$$

where c.c. denotes the complex conjugate of the preceding term and in the second equality we have defined $\delta \varphi_{1}=\frac{1}{\sqrt{2}}\left(\delta \psi^{1}+i \delta \psi^{2}\right)$ so that the indices $A, B$ run over real and imaginary parts. The kinetic term can be diagonalized by a rotation $\delta \psi^{A} \rightarrow R_{B}^{A} \delta \psi^{B}$. It is easy to verify that one eigenvalues of $K_{A B}$ is always negative and the Hamiltonian is unbounded below.

To summarize the results of this appendix: we have shown that a necessary (but not sufficient) condition for (34) to yield a perturbatively stable Hamiltonian is that the propagator $G\left(p^{2}\right) \sim 1 / F\left(-p^{2}\right)$ has at most a single pole. In this case the theory describes only a single degree of freedom and the equation of motion (38) requires only two initial data 11 Absent the imposition of some constraints (for example on the initial function $\phi_{0}(t)$ or following [43, 44, 45, 46]) ghost-like constituent excitations will lead to catastrophic classical instabilities and vacuum decay in the quantum theory. See [42] for stringent observational constraints on the existence of physical ghost fields.

There is one scenario where the analysis of this appendix can give very misleading results: the case where $\phi=0$ is a surface of degeneracy for the theory

\footnotetext{
${ }^{11}$ Stable theories describing multiple degrees of freedom can be constructed when the generatrix is not analytic, for example if $F(z)$ has isolated poles or branch cuts.
} 
(34). In this case the propagator obtained in a naive perturbation theory about $\phi=0$ will have less poles than the total number of physical degrees of freedom in the theory. This situation actually occurs for the level truncation of SFT, see appendix B for a review.

\section{APPENDIX C: Counting Initial Data for the SFT Tachyon}

It is sometime incorrectly argued that (55) admits only two initial conditions. This specious conclusion is reached by perturbing about the constant solution $\phi=0$, which corresponds to the unstable maximum of the potential. Writing $\phi=0+\delta \phi$ we have

$$
C^{-2 \square}(\square+1) \delta \phi=0
$$

to linear order in $\delta \phi$. The generatrix is $F(z)=C^{-2 z}(z+1)$ which has only a single zero corresponding to a tachyonic excitation with mass-squared $M^{2}=-1$ (in string units). At higher order in perturbation theory the counting of initial data is the same. However, this conclusion is very misleading. Notice that perturbing about the other critical point gives a different answer. Writing $\phi=C^{-3}+\delta \phi$ and linearizing in $\delta \phi$ we obtain

$$
\left[C^{-2 \square}(\square+1)-2\right] \delta \phi=0
$$

The generatrix is now $F(z)=C^{-2 z}(z+1)-2$ which has infinitely many zeroes $F\left(M_{n}^{2}\right)=0$ given by

$$
M_{n}^{2}=-\left[1+\frac{1}{2 \ln C} W_{n}\left(-\frac{4 \ln C}{C^{2}}\right)\right]
$$

where $n=0, \pm 1, \pm 2, \cdots$ and $W_{n}(x)$ denotes the branches of the Lambert-W function. The spectrum (C-3) corresponds to an infinite tower of constituent physical states with complex mass-squared. Correspondingly, equation (C-2) admits infinitely many initial conditions. In the Appendix of [28] we showed how to construct solutions of (55) near $\phi=0$ which depend on infinitely many free coefficients. Using the results of Appendix A, it follows that the Hamiltonian associated with (54) must be unbounded below.

Perturbing about different critical points of the potential we found different numbers of initial conditions. This is because $\phi=0$ is a surface of degeneracy for the equation of motion (55) [4]. This phenomenon has nothing to do with the nonlocal structure of the theory 12

\footnotetext{
${ }^{12} \mathrm{~A}$ simple example of a local theory with the same property was provided in [4]. Consider the Lagrangian $\mathcal{L}=-\frac{M^{3}}{2} \psi \ddot{\psi}^{2}$. The Euler-Lagrange equation is fourth order in time derivatives and hence generic solutions admit four initial data. However, on the surface $\psi=0$ the character of the equation of motion changes: instead of determining $\partial_{t}^{4} \psi$ in terms of lower order derivatives it instead becomes a constraint on the lower derivatives. Thus, solutions which start at the point $\psi=0$ contain only three free coefficients.
} 
The fact that (54) allows for negative kinetic energy cannot be doubted. Time-dependent solutions in flat space have been shown to move higher on the potential than their starting point, without difficulty [7]. Since energy is conserved in this rolling process, it follows that the kinetic energy must be negative. Moreover, generic solution of (55) undergo erratic oscillations with divergent frequency and amplitude, the hallmark behaviour of an Ostrogradskisick theory. The erratic time dependence of rolling tachyon solutions is properly understood as the symptom of a much deeper problem with SFT.

\section{References}

[1] W. Taylor, "Lectures on D-branes, tachyon condensation, and string field theory," arXiv:hep-th/0301094.

W. Taylor and B. Zwiebach, "D-branes, tachyons, and string field theory," arXiv:hep-th/0311017.

[2] M. R. Douglas and N. A. Nekrasov, "Noncommutative field theory," Rev. Mod. Phys. 73, 977 (2001) arXiv:hep-th/0106048.

R. J. Szabo, "Quantum Field Theory on Noncommutative Spaces," Phys. Rept. 378, 207 (2003) arXiv:hep-th/0109162.

[3] R. D. Sorkin, "Does locality fail at intermediate length-scales?," arXiv:gr-qc/0703099.

S. Hossenfelder, "A Note on Quantum Field Theories with a Minimal Length Scale," arXiv:0712.2811 [hep-th].

[4] D. A. Eliezer and R. P. Woodard, "The Problem of Nonlocality in String Theory," Nucl. Phys. B 325, 389 (1989).

[5] E. Witten, "Noncommutative Geometry And String Field Theory," Nucl. Phys. B 268, 253 (1986).

V. A. Kostelecky and S. Samuel, "The Static Tachyon Potential in the Open Bosonic String Theory," Phys. Lett. B 207, 169 (1988).

V. A. Kostelecky and S. Samuel, "On a Nonperturbative Vacuum for the Open Bosonic String," Nucl. Phys. B 336, 263 (1990)

I. Y. Aref'eva, A. S. Koshelev, D. M. Belov and P. B. Medvedev, "Tachyon condensation in cubic superstring field theory," Nucl. Phys. B 638, 3 (2002) arXiv:hep-th/0011117.

I. Y. Aref'eva, L. V. Joukovskaya and A. S. Koshelev, "Time evolution in superstring field theory on non-BPS brane. I: Rolling tachyon and energymomentum conservation," JHEP 0309, 012 (2003) arXiv:hep-th/0301137.

[6] P. G. O. Freund and M. Olson, "Nonarchimedean strings," Phys. Lett. B 199, 186 (1987).

P. G. O. Freund and E. Witten, "Adelic string amplitudes," Phys. Lett. B 199, 191 (1987).

L. Brekke, P. G. O. Freund, M. Olson and E. Witten, "Nonarchimedean String Dynamics," Nucl. Phys. B 302, 365 (1988). 
[7] N. Moeller and B. Zwiebach, "Dynamics with infinitely many time derivatives and rolling tachyons," JHEP 0210, 034 (2002) arXiv:hep-th/0207107.

[8] T. Biswas, M. Grisaru and W. Siegel, "Linear Regge trajectories from worldsheet lattice parton field theory," Nucl. Phys. B 708, 317 (2005) arXiv:hep-th/0409089.

D. Ghoshal, "p-adic string theories provide lattice discretization to the ordinary string worldsheet," Phys. Rev. Lett. 97, 151601 (2006).

[9] A. Sen, "Tachyon dynamics in open string theory," Int. J. Mod. Phys. A 20, 5513 (2005) arXiv:hep-th/0410103.

[10] N. Barnaby and J. M. Cline, "Creating the universe from brane-antibrane annihilation," Phys. Rev. D 70, 023506 (2004) arXiv:hep-th/0403223.

N. Barnaby, "Caustic formation in tachyon effective field theories," JHEP 0407, 025 (2004) arXiv:hep-th/0406120.

N. Barnaby, C. P. Burgess and J. M. Cline, "Warped reheating in braneantibrane inflation," JCAP 0504, 007 (2005) arXiv:hep-th/0412040.

N. Barnaby, A. Berndsen, J. M. Cline and H. Stoica, "Overproduction of cosmic superstrings," JHEP 0506, 075 (2005) arXiv:hep-th/0412095.

[11] G. R. Dvali and S. H. H. Tye, "Brane inflation," Phys. Lett. B 450, 72 (1999) arXiv:hep-ph/9812483.

C. P. Burgess, M. Majumdar, D. Nolte, F. Quevedo, G. Rajesh and R. J. Zhang, "The Inflationary Brane-Antibrane Universe," JHEP 0107, 047 (2001) arXiv:hep-th/0105204.

S. Kachru, R. Kallosh, A. D. Linde, J. M. Maldacena, L. P. McAllister and S. P. Trivedi, "Towards inflation in string theory," JCAP 0310, 013 (2003) arXiv:hep-th/0308055.

D. Baumann, A. Dymarsky, I. R. Klebanov, L. McAllister and P. J. Steinhardt, "A Delicate Universe," Phys. Rev. Lett. 99, 141601 (2007) arXiv:0705.3837 [hep-th]].

[12] A. Pais and G. E. Uhlenbeck, "On Field theories with nonlocalized action," Phys. Rev. 79, 145 (1950).

[13] D. Evens, J. W. Moffat, G. Kleppe and R. P. Woodard, "Nonlocal regularizations of gauge theories," Phys. Rev. D 43, 499 (1991).

[14] S. Deser and R. P. Woodard, "Nonlocal Cosmology," Phys. Rev. Lett. 99, 111301 (2007) arXiv:0706.2151 [astro-ph]].

[15] T. Koivisto, "Dynamics of Nonlocal Cosmology," Phys. Rev. D 77, 123513 (2008) arXiv:0803.3399 [gr-qc]].

T. S. Koivisto, "Newtonian limit of nonlocal cosmology," Phys. Rev. D 78, 123505 (2008) [arXiv:0807.3778 [gr-qc]]. 
[16] I. Y. Aref'eva, "Nonlocal string tachyon as a model for cosmological dark energy," AIP Conf. Proc. 826, 301 (2006) arXiv:astro-ph/0410443.

I. Y. Aref'eva and L. V. Joukovskaya, "Time lumps in nonlocal stringy models and cosmological applications," JHEP 0510, 087 (2005) arXiv:hep-th/0504200.

I. Y. Aref'eva, A. S. Koshelev and S. Y. Vernov, "Stringy dark energy model with cold dark matter," Phys. Lett. B 628, 1 (2005) arXiv:astro-ph/0505605.

I. Y. Aref'eva, A. S. Koshelev and S. Y. Vernov, "Crossing of the $\mathrm{w}=-1$ barrier by D3-brane dark energy model," Phys. Rev. D 72, 064017 (2005) arXiv:astro-ph/0507067.

I. Y. Aref'eva and A. S. Koshelev, "Cosmic acceleration and crossing of w $=-1$ barrier from cubic superstring field theory," arXiv:hep-th/0605085.

I. Y. Aref'eva and I. V. Volovich, "On the null energy condition and cosmology," arXiv:hep-th/0612098.

I. Y. Aref'eva, L. V. Joukovskaya and S. Y. Vernov, "Bouncing and accelerating solutions in nonlocal stringy models," arXiv:hep-th/0701184.

[17] J. E. Lidsey, "Stretching the inflaton potential with kinetic energy," arXiv:hep-th/0703007.

[18] D. J. Mulryne and N. J. Nunes, "Diffusing non-local inflation: Solving the field equations as an initial value problem," Phys. Rev. D 78, 063519 (2008) arXiv:0805.0449 [hep-th]].

N. J. Nunes and D. J. Mulryne, "Non-linear non-local Cosmology," AIP Conf. Proc. 1115, 329 (2009) arXiv:0810.5471 [astro-ph]].

[19] T. Biswas, A. Mazumdar and W. Siegel, "Bouncing universes in stringinspired gravity," JCAP 0603, 009 (2006) arXiv:hep-th/0508194.

T. Biswas, R. Brandenberger, A. Mazumdar and W. Siegel, "Nonperturbative gravity, Hagedorn bounce and CMB," arXiv:hep-th/0610274 T. Biswas, T. Koivisto and A. Mazumdar, "Resolution of the Cosmological Singularity in Non-local Higher Derivative Theories of Gravity," arXiv:1005.0590 [hep-th].

[20] J. Khoury, "Fading gravity and self-inflation," arXiv:hep-th/0612052.

[21] N. Barnaby, T. Biswas and J. M. Cline, "p-adic inflation," JHEP 0704, 056 (2007) arXiv:hep-th/0612230.

[22] N. Barnaby and J. M. Cline, "Large Nongaussianity from Nonlocal Inflation," JCAP 0707, 017 (2007) [arXiv:0704.3426 [hep-th]].

[23] N. Barnaby and J. M. Cline, "Predictions for Nongaussianity from Nonlocal Inflation," JCAP 0806, 030 (2008) arXiv:0802.3218 [hep-th]].

[24] N. Barnaby and N. Kamran, "Dynamics with Infinitely Many Derivatives: The Initial Value Problem," JHEP 0802, 008 (2008) arXiv:0709.3968 [hepth]]. 
[25] N. Barnaby, "Nonlocal Inflation," Can. J. Phys. 87, 189 (2009) arXiv:0811.0814 [hep-th]].

[26] M. Kiermaier, Y. Okawa and B. Zwiebach, "The boundary state from open string fields," arXiv:0810.1737 [hep-th].

[27] S. Hellerman and M. Schnabl, "Light-like tachyon condensation in Open String Field Theory," arXiv:0803.1184 [hep-th].

[28] N. Barnaby, D. J. Mulryne, N. J. Nunes and P. Robinson, "Dynamics and Stability of Light-Like Tachyon Condensation," JHEP 0903, 018 (2009) arXiv:0811.0608 [hep-th]].

[29] F. Beaujean and N. Moeller, "Delays in Open String Field Theory," arXiv:0912.1232 [hep-th].

[30] L. Joukovskaya, "Dynamics with Infinitely Many Time Derivatives in Friedmann-Robertson-Walker Background and Rolling Tachyon," JHEP 0902, 045 (2009) arXiv:0807.2065 [hep-th]].

[31] N. Barnaby and N. Kamran, "Dynamics with Infinitely Many Derivatives: Variable Coefficient Equations," JHEP 0812, 022 (2008) arXiv:0809.4513 [hep-th]].

[32] J. Gomis, K. Kamimura and J. Llosa, "Hamiltonian formalism for space-time non-commutative theories," Phys. Rev. D 63, 045003 (2001) arXiv:hep-th/0006235.

J. Gomis, K. Kamimura and T. Ramirez, "Physical reduced phase space of non-local theories," Nucl. Phys. B 696, 263 (2004) arXiv:hep-th/0311184.

[33] R. P. Woodard, "Avoiding dark energy with 1/R modifications of gravity," arXiv:astro-ph/0601672.

[34] D. A. Eliezer and R. P. Woodard, "Instability of higher difference initial value theories," Phys. Rev. D 40, 465 (1989).

G. Kleppe and R. P. Woodard, "A Supersymmetric regulator," Phys. Lett. B 253, 331 (1991).

G. Kleppe and R. P. Woodard, "Two loop calculations using nonlocal regularization," Annals Phys. 221, 106 (1993).

G. Kleppe and R. P. Woodard, "Nonlocal Yang-Mills," Nucl. Phys. B 388, 81 (1992) arXiv:hep-th/9203016.

D. L. Bennett, H. B. Nielsen and R. P. Woodard, "The initial value problem for maximally non-local actions," Phys. Rev. D 57, 1167 (1998) arXiv:hep-th/9707088.

R. P. Woodard, "A canonical formalism for Lagrangians with nonlocality of finite extent," Phys. Rev. A 62, 052105 (2000) arXiv:hep-th/0006207. R. P. Woodard, "The Ostrogradskian instability of Lagrangians with nonlocality of finite extent," Phys. Rev. A 67, 016102 (2003) arXiv:hep-th/0207191. 
M. E. Soussa and R. P. Woodard, "A Nonlocal Metric Formulation of MOND," Class. Quant. Grav. 20, 2737 (2003) arXiv:astro-ph/0302030.

[35] G. Calcagni, M. Montobbio and G. Nardelli, "Localization of nonlocal theories," Phys. Lett. B 662, 285 (2008) [arXiv:0712.2237 [hep-th]].

[36] T. Biswas, J. A. R. Cembranos and J. I. Kapusta, "Thermal Duality and Hagedorn Transition from p-adic Strings," Phys. Rev. Lett. 104, 021601 (2010) arXiv:0910.2274 [hep-th]].

[37] T. Biswas, J. A. R. Cembranos and J. I. Kapusta, "Thermodynamics and Cosmological Constant of Non-Local Field Theories from p-Adic Strings," arXiv:1005.0430 [hep-th].

[38] M. Gutperle and A. Strominger, "Spacelike branes," JHEP 0204, 018 (2002) arXiv:hep-th/0202210.

[39] D. Kutasov, M. Marino and G. W. Moore, "Some exact results on tachyon condensation in string field theory," JHEP 0010, 045 (2000) arXiv:hep-th/0009148.

[40] N. D. Lambert, H. Liu and J. M. Maldacena, "Closed strings from decaying D-branes," JHEP 0703, 014 (2007) arXiv:hep-th/0303139.

[41] A. Sen, "Open and closed strings from unstable D-branes," Phys. Rev. D 68, 106003 (2003) arXiv:hep-th/0305011.

A. Sen, "Open-closed duality at tree level," Phys. Rev. Lett. 91, 181601 (2003) arXiv:hep-th/0306137.

[42] J. M. Cline, S. Jeon and G. D. Moore, "The phantom menaced: Constraints on low-energy effective ghosts," Phys. Rev. D 70, 043543 (2004) arXiv:hep-ph/0311312.

[43] T. D. Lee and G. C. Wick, "Negative Metric and the Unitarity of the S Matrix," Nucl. Phys. B 9, 209 (1969).

T. D. Lee and G. C. Wick, "Finite Theory of Quantum Electrodynamics," Phys. Rev. D 2, 1033 (1970).

[44] B. Grinstein, D. O'Connell and M. B. Wise, "The Lee-Wick standard model," Phys. Rev. D 77, 025012 (2008) arXiv:0704.1845 [hep-ph]].

[45] R. E. Cutkosky, P. V. Landshoff, D. I. Olive and J. C. Polkinghorne, "A non-analytic S matrix," Nucl. Phys. B 12, 281 (1969).

[46] S. W. Hawking and T. Hertog, "Living with ghosts," Phys. Rev. D 65, 103515 (2002) arXiv:hep-th/0107088.

[47] B. Grinstein, D. O'Connell and M. B. Wise, "Causality as an emergent macroscopic phenomenon: The Lee-Wick $\mathrm{O}(\mathrm{N})$ model," arXiv:0805.2156 [hep-th]. 
[48] L. Kofman, A. D. Linde and A. A. Starobinsky, "Towards the theory of reheating after inflation," Phys. Rev. D 56, 3258 (1997) arXiv:hep-ph/9704452.

[49] A. Sen, "Tachyon matter," JHEP 0207, 065 (2002) arXiv:hep-th/0203265. A. Sen, "Field theory of tachyon matter," Mod. Phys. Lett. A 17, 1797 (2002) arXiv:hep-th/0204143.

[50] T. Erler and D. J. Gross, "Locality, causality, and an initial value formulation for open string field theory," arXiv:hep-th/0406199.

[51] T. Erler, "Level truncation and rolling the tachyon in the lightcone basis for open string field theory," arXiv:hep-th/0409179.

[52] E. Coletti, I. Sigalov and W. Taylor, "Taming the tachyon in cubic string field theory," JHEP 0508, 104 (2005) arXiv:hep-th/0505031.

[53] K. Marvel and D. Wesley, "Tunneling with negative tension," JHEP 0812, 034 (2008) [arXiv:0808.3186 [hep-th]].

[54] E. I. Buchbinder, J. Khoury and B. A. Ovrut, "New Ekpyrotic Cosmology," Phys. Rev. D 76, 123503 (2007) arXiv:hep-th/0702154. 


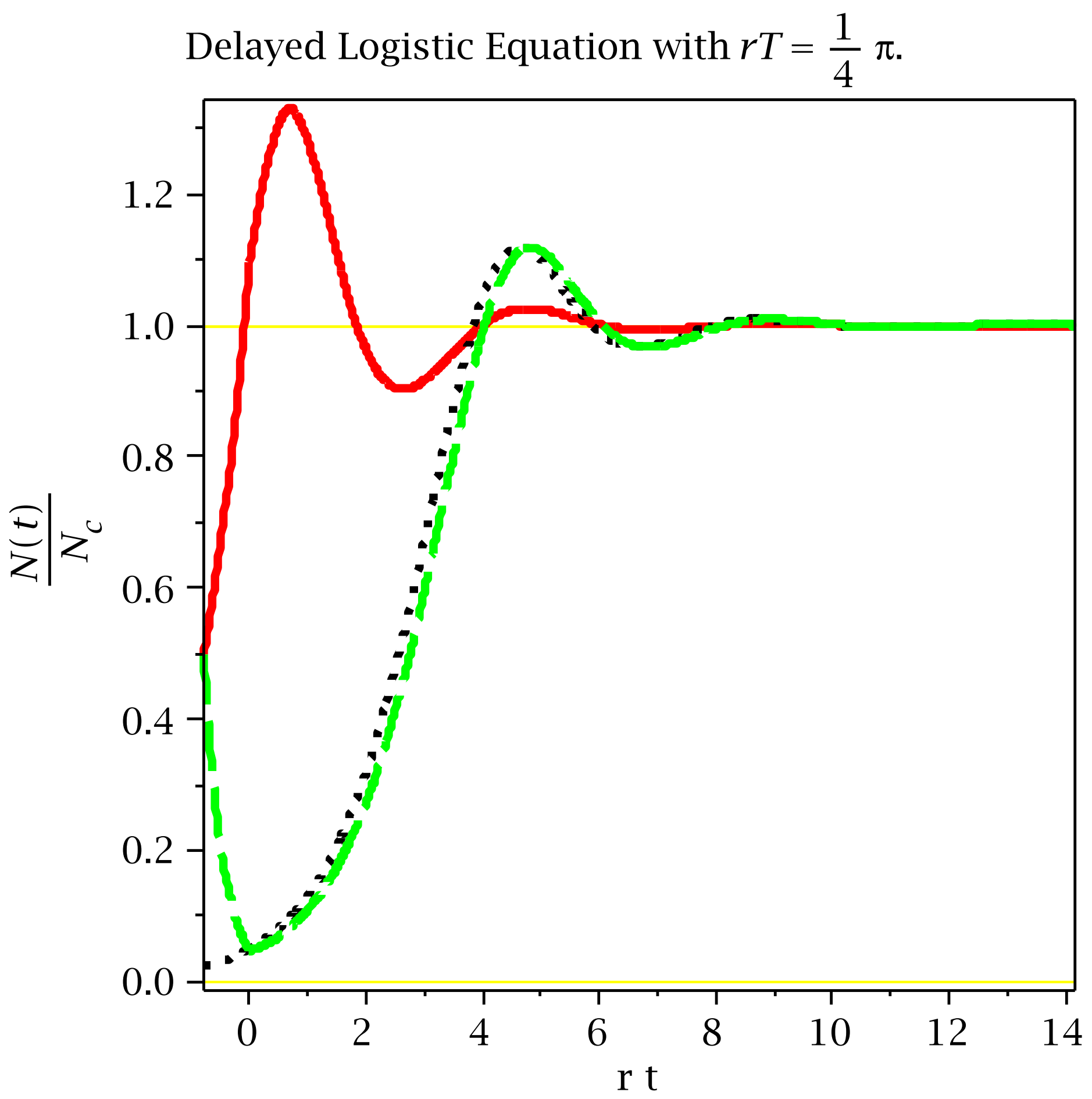




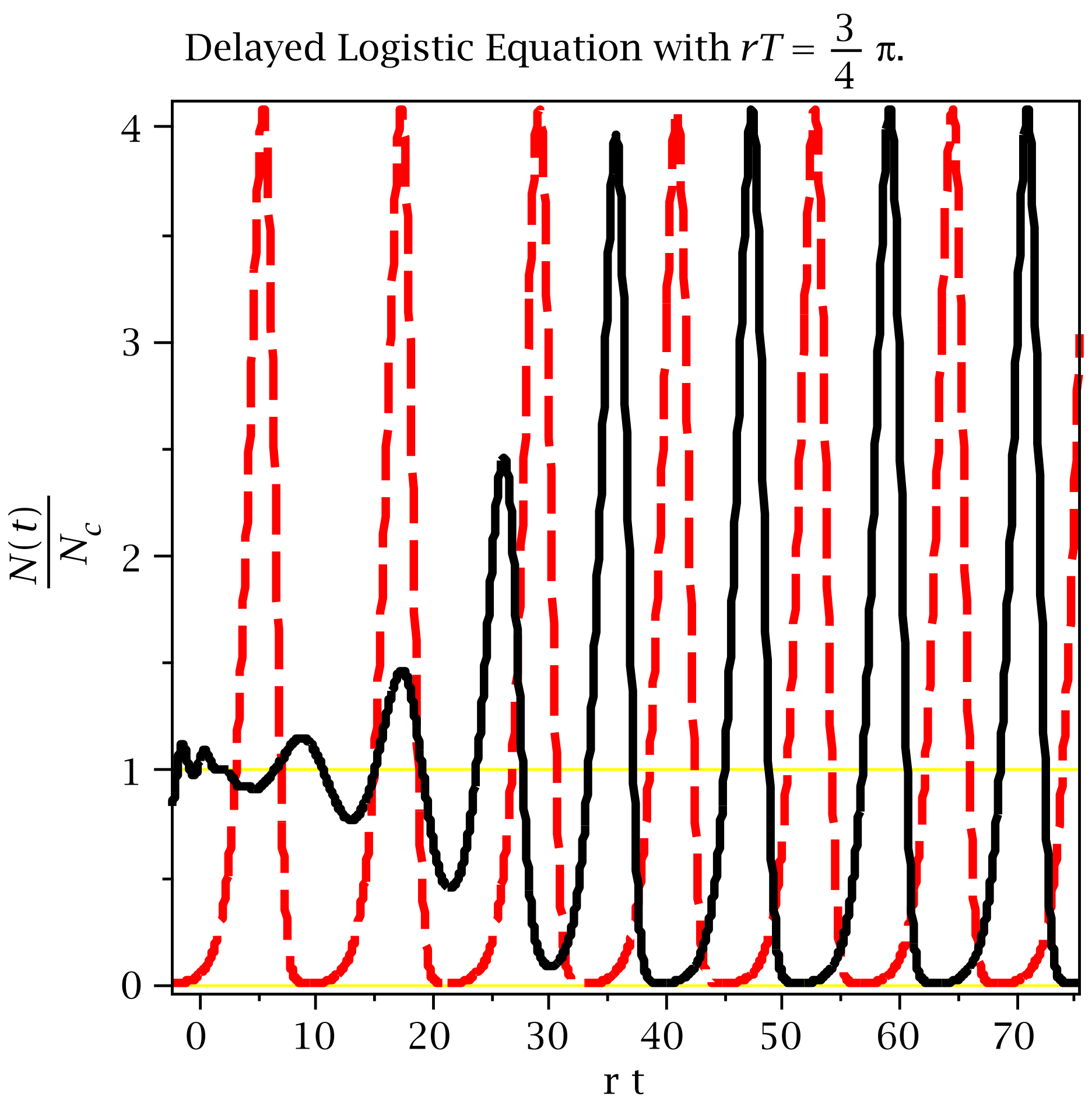




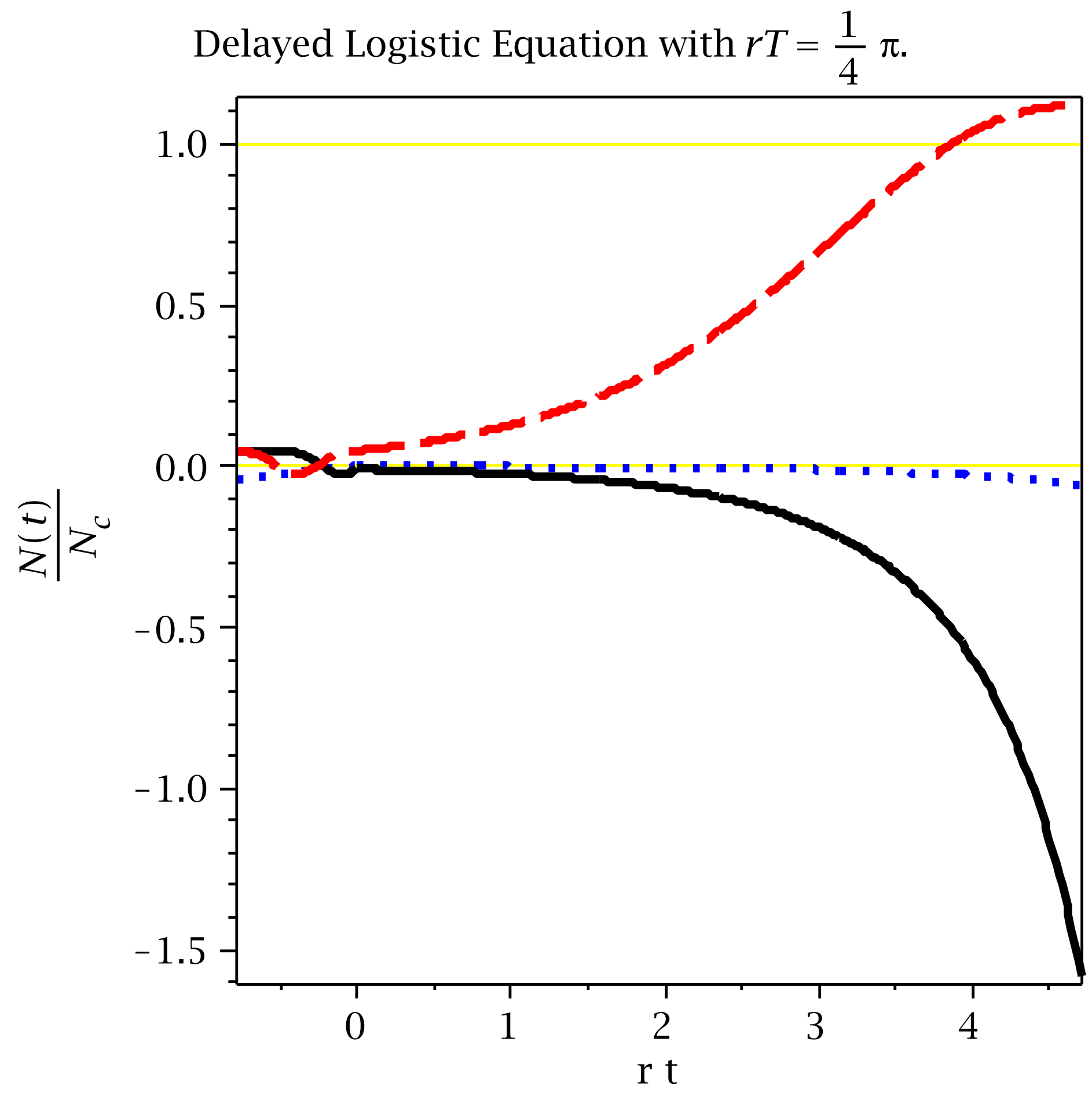

